\title{
Spectra and structural polynomials of graphs of relevance to the theory of molecular conduction
}

\author{
Patrick W. Fowler *, Barry T. Pickup \\ Department of Chemistry, University of Sheffield, Sheffield, S3 7HF, UK \\ Irene Sciriha \\ Department of Mathematics, University of Malta, Msida, Malta \\ Martha Borg \\ Department of Chemistry, University of Sheffield, Sheffield, S3 7HF, UK
}

Received 3 November 2016, accepted 28 February 2017, published online 24 March 2017

\begin{abstract}
In chemistry and physics, distortivity of $\pi$-systems (stabilisation of bond-alternated structures) is an important factor in the calculation of geometric, energetic, and electronic properties of molecules via graph theoretical methods. We use the spectra of paths and cycles with alternating vertex and edge weights to obtain the eigenvalues and eigenvectors for a class of linear and cyclic ladders with alternating rung and backbone edge weights. We derive characteristic polynomials and other structural polynomials formed from the cofactors of the characteristic matrix for these graphs. We also obtain spectra and structural polynomials for ladders with flipped weights and/or Möbius topology. In all cases, the structural polynomials for the composite graphs are expressed in terms of products of polynomials for graphs of half order. This form of the expressions allows global deductions about the transmission spectra of molecular devices in the graph-theoretical theory of ballistic molecular conduction.
\end{abstract}

Keywords: Adjacency matrix, characteristic polynomial, molecular conduction, eigenvalues, weighted graphs.

Math. Subj. Class.: 05C50

${ }^{*}$ Corresponding author.

E-mail addresses: p.w.fowler@sheffield.ac.uk (Patrick W. Fowler), b.t.pickup@ sheffield.ac.uk (Barry T. Pickup), irene.sciriha-aquilina@um.edu.mt (Irene Sciriha), mborg1@sheffield.ac.uk (Martha Borg) 


\section{Introduction}

Our aim is to use weighting of graphs as a tool for the study of ballistic molecular conduction in undistorted and distorted molecular and extended systems. In this article we derive the spectra and characteristic polynomials of a series of graphs that possess three common features. The first is that they are bipartite. The second is that they possess an involution that allows the graph to be expressed as a product of simpler graphs with known spectra. The third feature is termed 'distortivity' by physical scientists. This refers to the way that the spectrum changes with edge weights, and is of prime importance in theories of electronic structure, where molecular structures are modelled by graphs. It is well known to physicists and chemists that extended overlapping $\pi$-electron systems may achieve greater stability by distorting in such a way that bond lengths alternate, and the sharing of electron density across the $\pi$-system is reduced. This is known in the physics literature as Peierls distortion [13], and in the chemical literature as Jahn-Teller distortion [12]. It typically affects $\pi$-electron systems in such a way as to reduce their conductivity. In order to assess the importance of distortivity for the specific phenomenon of ballistic molecular conduction, we need explicit characteristic polynomials and spectra for families of weighted graphs representing molecules of chemical interest.

\subsection{Graph theoretical background}

The graphs in which we are interested are linear ladders, their cyclic analogues the treadmills, and graphs derivable from them by using (signed or zero) weights, such as linear polyacenes and (Möbius) cyclacenes, shown in Fig. 1. In graph theory terms, we can mimic

(a)

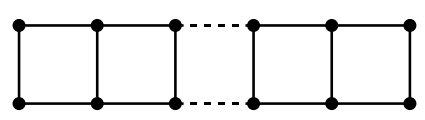

(c)

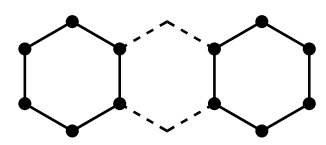

(b)

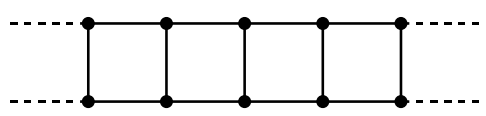

(d)

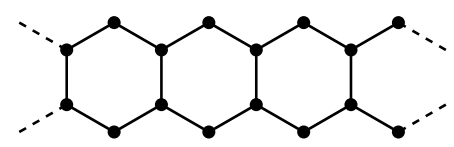

Figure 1: Families of graphs treated in this paper: (a) ladders; (b) treadmills; (c) linear polyacenes; (d) cyclic polyacenes.

geometric distortion of a molecular framework by studying weighted graphs in which edge weights alternate [11]. Adjacency matrices of such graphs have been studied by Gover [9] in the form of 2-Toeplitz matrices. Gover gave an explicit solution for the spectra of 2-Toeplitz matrices of odd dimension, and an implicit solution for even dimensions. These solutions form the basis for our treatment of ladders and treadmills. Ladders (treadmills) comprise two backbone chains (rings), that are linked by 'rungs'. We shall alternate the weights on the rungs, and separately on the edges comprising the two backbones, in such a way that an involution symmetry is preserved. This symmetry element swaps vertices in upper and lower backbone chains of the graphs and is crucial for the solution of the secular problem for distorted and undistorted systems. The use of symmetry splits the secular matrices of the graphs into two non-interacting blocks, each of which represents a single 
path (or cycle) with alternating weighted vertices and edges. It is these backbone graphs that possess the analytical solutions derived previously by Gover [9] and Shin [18].

An active avenue of research is exploration of the influence of molecular topology in conduction behaviour. We therefore include certain graphs with edge weights having flipped signs, and/or with a pair of crossed backbone edges. These flipped and crossed graphs are sometimes called Möbius graphs [6]. The cases that we consider here have closed-form spectra and structural polynomials that can be derived using the methodology used for unflipped, uncrossed graphs.

\subsection{Physical motivation}

The physical context for the present mathematical exploration is that electronic structure of unsaturated carbon networks is qualitatively modelled using spectral graph theory. In particular, the basic reason for our interest in the graphs described in this paper is our research into molecular conductivity in small molecules [14, 15] using the source-sinkpotential (SSP) method of Ernzerhof et al. [3, 4, 5, 20]. This approach uses graph theory as a vehicle for showing important qualitative features in electron transmission for individual molecules. Central to the SSP method is the idea of a molecular device based on a molecular graph, in which the effects of infinite attached wires are represented by two special extra vertices, which behave respectively as a source and sink (of electrons). We have shown [14] that electronic transmission in this model can be expressed using a basic set of polynomials related to the molecular graph, $\mathcal{G}$. These are the characteristic polynomial,

$$
s(E)=\operatorname{det}(E \mathbf{1}-\mathbf{A})
$$

and the cofactors of the characteristic matrix,

$$
\jmath_{\mathrm{pq}}(E)=(-1)^{\mathrm{p}+\mathrm{q}} \operatorname{det}(E \mathbf{1}-\mathbf{A})^{[\mathrm{p}, \mathrm{q}]}=(E \mathbf{1}-\mathbf{A})_{\mathrm{pq}}^{-1} s(E),
$$

where $E$ is the energy of the transmitted electron, $\mathbf{1}$ is the $n \times n$ unit matrix and $\mathbf{A}$ is the $n \times n$ adjacency matrix of the graph $\mathcal{G}$ of order $n$. The indices in square-brackets refer to the sets of rows and columns deleted from the determinant of the characteristic matrix.

The eigenvalue problem,

$$
\mathbf{A c}_{\mathrm{k}}=\mathbf{c}_{\mathrm{k}} \epsilon_{\mathrm{k}}
$$

allows us to define the $n$ eigenvalues $\left\{\epsilon_{\mathrm{k}}\right\}$, and the corresponding eigenvectors $\mathbf{c}_{\mathrm{k}}$. Spectral decomposition allows us to write

$$
s(E)=\prod_{\mathrm{k}=1}^{n}\left(E-\epsilon_{\mathrm{k}}\right),
$$

and spectral resolution of the inverse gives a general expression for all $\jmath_{\mathrm{pq}}(E)$ polynomials in terms of eigenvectors and eigenvalues of $\mathbf{A}$ :

$$
\jmath_{\mathrm{pq}}(E)=\sum_{\mathrm{k}=1}^{n} \frac{c_{\mathrm{pk}} c_{\mathrm{qk}}}{E-\epsilon_{\mathrm{k}}}
$$

where $c_{\mathrm{pk}}$ is the pth entry in the kth eigenvector, $\mathbf{c}_{\mathrm{k}}$. In what follows we will find it useful to switch between the two approaches, viz. calculating structural polynomials from 
determinants of characteristic matrices, or from explicit solutions of the eigenvalue problem Eq. (1.3).

For a specific device in which vertices $\mathrm{p}$ and $\mathrm{q}$ of the molecular graph are attached to infinite conducting wires, one needs just four polynomials, namely, $s, \jmath_{\mathrm{pp}}, \jmath_{\mathrm{qq}}$, and

$$
v_{\mathrm{pq}, \mathrm{pq}}(E)=\operatorname{det}(E \mathbf{1}-\mathbf{A})^{[\mathrm{pq}, \mathrm{pq}]}
$$

to deduce an expression for the transmission, $T(E)$, of an incoming stream of electrons [14]. These are all characteristic polynomials derived from vertex-deleted graphs:

$$
\begin{aligned}
s & \equiv \varphi(\mathcal{G}, E), \\
t & \equiv \jmath_{\mathrm{pp}}=\varphi(\mathcal{G}-\mathrm{p}, E), \\
u & \equiv \jmath_{\mathrm{qq}}=\varphi(\mathcal{G}-\mathrm{q}, E), \\
v & \equiv v_{\mathrm{pq}, \mathrm{pq}}(E)=\varphi(\mathcal{G}-\mathrm{p}-\mathrm{q}, E),
\end{aligned}
$$

where $\varphi(\mathcal{G}, E)$ is the characteristic polynomial of graph $\mathcal{G}$, and the letters $s, t, u$, and $v$ refer to literature notation [14]. The formula for $v_{\mathrm{pq}, \mathrm{pq}}$ can be deduced using Jacobi's relation [19]:

$$
s v_{\mathrm{pq}, \mathrm{pq}}=\jmath_{\mathrm{pp}} \jmath_{\mathrm{qq}}-\jmath_{\mathrm{pq}}^{2} .
$$

For convenience, we refer to $s(E)$, the $\jmath_{\mathrm{pq}}(E)$ and $v(E)$ as structural polynomials.

We have shown [15] that molecular conduction can be thought of in two different ways, i.e. either as occurring through molecular bonds (graph edges), or through individual molecular orbitals (eigenvectors of the graph adjacency matrix). We find that there are 11 basic categories of conduction $[7,15,17]$ for molecules and that these are determined by the eigenvector coefficients. Conduction behaviour at eigenvalues of the adjacency matrix is particularly important [15]. Hence arises our interest in closed-form expressions for spectra and structural polynomials. Spectral representations of the structural polynomials are also informative, in that they allow elaboration of the SSP model to treat the physically important effects of Pauli exclusion, an effect that prevents current passing through filled orbitals. This extension of the theory is worked out in a recent paper [16].

We can summarise the key features of our approach and the main results as follows. Explicit expressions for structural polynomials, spectra and eigenvectors of weighted paths and cycles are obtained. These are useful in themselves for the discussion of distortivity and conduction. We then exploit the graph-product structure of the families of ladders, treadmills and Möbius forms to build analytical expressions for the structural polynomials and spectral properties of these graphs in terms of those of the simpler graphs. This gives compact formulas that are ultimately related to Chebyshev and similar orthogonal polynomials. It is this 'factorised' form of the final expressions that gives a powerful tool for interpretation of spectra and conduction properties of ladders, treadmills. This interpretation will be used to analyse the effects of flips and twists on conduction in physically realisable systems.

\subsection{Plan}

The plan of the paper is as follows. First we derive eigenvectors and eigenvalues for weighted alternating paths (Section 2), and then derive expressions for the important structural polynomials in Section 3. These results are used to derive spectra for ladders and 
their structural polynomials in Sections 4 and 5. The spectra for alternating cycles are derived in Section 6, and their structural polynomials in Section 7. Derivations of spectra and structural polynomials of treadmills then follow in Sections 8 and 9. Section 10 introduces important chemical graphs that can be derived from ladders and treadmills. We end with a brief conclusion.

Our explicit treatment of cases necessarily leads to a large number of equations, but the central results are Eqs. (5.6) and (9.3), which show the generic relationships between the structural polynomials of ladders and chains, and treadmills and cycles, respectively. The structural polynomials for weighted chains are given in Eqs. (3.12) and (3.13), and weighted cycles in Eqs. (7.13) and (7.14) and for flipped cycles in Eqs. (7.15) and (7.16). The blocks of equations giving the results are: Eqs. (5.7) to (5.10) for ladders; Eqs. (9.4) and (9.5) for treadmills; Eqs. (9.9) and (9.10) for flipped treadmills; Eqs. (9.14) and (9.15) for Möbius treadmills; Eqs. (9.19) and (9.20) for flipped Möbius treadmills.

\section{The spectra of alternating weighted paths $P_{M}(a, b \mid c, d)$}

We consider paths, $P_{M}(a, b \mid c, d)$, with alternating vertex weights $a, b$, and edge weights $c, d$. Eigenvalues and eigenvectors for such weighted paths have been deduced by Gover [9] and Shin [18]. Gover used recursion to show that the spectrum of the odd-vertex chain, $P_{2 N+1}$, could be expressed in terms of two sets of polynomials. One is the Chebyshev polynomials of the second kind, $U_{N}$. The other set of polynomials satisfy the Chebyshev recursion relation, but with different initial values. The eigenvectors for the odd paths are evaluated at the zeroes of the polynomial $U_{N}$. The even paths have an analogous form for eigenvectors and eigenvalues, but one of the quantities cannot be evaluated analytically. We discuss odd and even paths separately.

\subsection{The odd path, $P_{2 N+1}(a, b \mid c, d)$}

A path, $P_{2 N+1}(a, b \mid c, d)$, with $2 N+1$ vertices is shown in Fig. 2. It is convenient to write

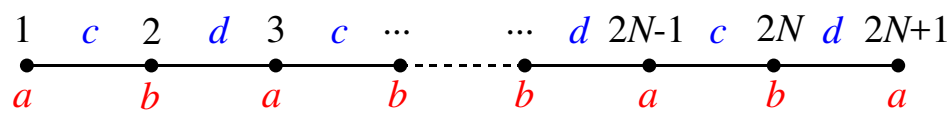

Figure 2: A chain, $P_{2 N+1}(a, b \mid c, d)$, with $2 N+1$ vertices and alternating vertex weights $(a, b)$, and edge weights $(c, d)$.

the adjacency matrix, $\mathbf{A}^{\mathrm{P}}$, for this bipartite graph in the form

$$
\mathbf{A}^{\mathrm{P}}=\left(\begin{array}{cc}
a \mathbf{1}_{N+1} & \mathbf{B}^{\mathrm{P}} \\
\left(\mathbf{B}^{\mathrm{P}}\right)^{\mathrm{T}} & b \mathbf{1}_{N}
\end{array}\right),
$$

where $\mathbf{1}_{h}$ symbolises a unit matrix of dimension $h$, and superscript $\mathrm{T}$ indicates a transpose. We place the $(N+1)$ odd-numbered vertices shown in Fig. 2 in the first block, and the $N$ 
even-numbered vertices in the second. The $(N+1) \times N$-dimensional matrix $\mathbf{B}^{\mathrm{P}}$ is then

$$
\mathbf{B}^{\mathrm{P}}=\left(\begin{array}{ccccc}
c & 0 & \cdots & 0 & 0 \\
d & c & \ddots & & 0 \\
0 & \ddots & \ddots & \ddots & \vdots \\
\vdots & \ddots & \ddots & \ddots & 0 \\
0 & & \ddots & d & c \\
0 & 0 & \cdots & 0 & d
\end{array}\right) .
$$

In order to find the eigenvalues of the matrix $\mathbf{A}^{\mathrm{P}}$ we can use the fact that the blocks on the diagonal are invariant to any unitary transformation. Therefore, a singular value decomposition of the off diagonal block will render the whole matrix in a form in which each block is diagonal or pseudo-diagonal. This technique has been used [15], for example, to provide a compact derivation of the Coulson-Rushbrooke theorem for bipartite graphs [1]. The singular value decomposition [8, Sections 2.5.3 and 2.5.6] of $\mathbf{B}^{\mathrm{P}}$ can be written as

$$
\mathbf{B}^{\mathrm{P}} \mathbf{X}^{\mathrm{P}}=\mathbf{Y}^{\mathrm{P}} \boldsymbol{\sigma}^{\mathrm{P}}
$$

where $\mathbf{X}^{\mathrm{P}}$, and $\mathrm{Y}^{\mathrm{P}}$ are $N$ - and $(N+1)$-dimensional orthogonal matrices, respectively. The $(N+1) \times N$-dimensional rectangular matrix, $\boldsymbol{\sigma}^{\mathrm{P}}$, is "diagonal", i.e.

$$
\left(\begin{array}{cccc}
\sigma_{1}^{\mathrm{P}} & 0 & \cdots & 0 \\
0 & \sigma_{2}^{\mathrm{P}} & \ddots & \vdots \\
\vdots & \ddots & \ddots & 0 \\
0 & & \ddots & \sigma_{N}^{\mathrm{P}} \\
0 & 0 & \cdots & 0
\end{array}\right)
$$

and the singular values $\sigma_{\mathrm{k}}^{\mathrm{P}} \geq 0$, have labels $\mathrm{k}$. The theory of singular value decomposition tells us further that

$$
\begin{array}{cl}
\left(\mathbf{B}^{\mathrm{P}}\right)^{\mathrm{T}} \mathbf{B}^{\mathrm{P}} \mathbf{X}_{k}^{\mathrm{P}}=\mathbf{X}_{\mathrm{k}}^{\mathrm{P}}\left(\sigma_{\mathrm{k}}^{\mathrm{P}}\right)^{2} & \text { for } \mathrm{k}=1, \ldots, N, \\
\mathbf{B}^{\mathrm{P}}\left(\mathbf{B}^{\mathrm{P}}\right)^{\mathrm{T}} \mathbf{Y}_{\mathrm{k}}^{\mathrm{P}}=\mathbf{Y}_{\mathrm{k}}^{\mathrm{P}}\left(\sigma_{\mathrm{k}}^{\mathrm{P}}\right)^{2} & \text { for } \mathrm{k}=1, \ldots, N+1,
\end{array}
$$

with $\sigma_{\mathrm{k}}^{\mathrm{P}}>0$ for $\mathrm{k}=1, \ldots, N$, and $\sigma_{N+1}^{\mathrm{P}}=0$. We note that the $N \times N$-dimensional positive definite tridiagonal matrix

$$
\left(\mathbf{B}^{\mathrm{P}}\right)^{\mathrm{T}} \mathbf{B}^{\mathrm{P}}=\left(\begin{array}{cccccc}
c^{2}+d^{2} & c d & 0 & \cdots & 0 & 0 \\
c d & c^{2}+d^{2} & c d & \ddots & & 0 \\
0 & c d & c^{2}+d^{2} & \ddots & \ddots & \vdots \\
\vdots & \ddots & \ddots & \ddots & \ddots & 0 \\
0 & & \ddots & \ddots & c^{2}+d^{2} & c d \\
0 & 0 & \cdots & 0 & c d & c^{2}+d^{2}
\end{array}\right)
$$


represents the adjacency matrix of a path of length $N$ with equal vertex weights $\left(c^{2}+d^{2}\right)$, and equal edge weights $c d$, so the eigenvalues are

$$
\left(\sigma_{\mathrm{k}}^{\mathrm{P}}\right)^{2}=c^{2}+d^{2}+2 c d \cos \theta_{\mathrm{k}}^{\mathrm{P}} \quad \text { for } \mathrm{k}=1,2, \ldots, N,
$$

where the angle

$$
\theta_{\mathrm{k}}^{\mathrm{P}}=\frac{\pi \mathrm{k}}{N+1}
$$

also describes the orthonormal eigenvectors

$$
X_{\mathrm{pk}}^{\mathrm{P}}=N_{\mathrm{k}}^{\mathrm{P}} \sin \mathrm{p} \theta_{\mathrm{k}}^{\mathrm{P}},
$$

and the normalisation factor is

$$
N_{\mathrm{k}}^{\mathrm{P}}=\sqrt{\frac{2}{N+1}} .
$$

The $(N+1) \times(N+1)$-dimensional semi-definite tridiagonal matrix, on the other hand,

$$
\mathbf{B}^{\mathrm{P}}\left(\mathbf{B}^{\mathrm{P}}\right)^{\mathrm{T}}=\left(\begin{array}{cccccc}
c^{2} & c d & 0 & \cdots & 0 & 0 \\
c d & c^{2}+d^{2} & c d & \ddots & & 0 \\
0 & c d & c^{2}+d^{2} & \ddots & \ddots & \vdots \\
\vdots & \ddots & \ddots & \ddots & \ddots & 0 \\
0 & & \ddots & \ddots & c^{2}+d^{2} & c d \\
0 & 0 & \cdots & 0 & c d & d^{2}
\end{array}\right),
$$

has no such simple expressions for its eigenvectors, but they can be derived directly from the singular value decomposition. We can use Eq. (2.3) to deduce that

$$
Y_{\mathrm{pk}}^{\mathrm{P}}=\frac{1}{\sigma_{\mathrm{k}}^{\mathrm{P}}}\left(c X_{\mathrm{pk}}^{\mathrm{P}}+d X_{\mathrm{p}-1, \mathrm{k}}^{\mathrm{P}}\right) \quad \text { for } \mathrm{k}, \mathrm{p}=1,2, \ldots, N .
$$

We note from Eq. (2.9) that $\mathrm{p}=0$ implies $X_{0 \mathrm{k}}^{\mathrm{P}}=0$, and $\mathrm{p}=N+1$, implies $X_{N+1, \mathrm{k}}^{\mathrm{P}}=0$. The nullspace vector is

$$
Y_{\mathrm{p}, N+1}^{\mathrm{P}}=N_{N+1}^{\mathrm{P}}(-1)^{\mathrm{p}-1} c^{\mathrm{p}-1} d^{N-\mathrm{p}+1},
$$

where the normalisation factor is

$$
N_{N+1}^{\mathrm{P}}=\sqrt{\frac{d^{2}-c^{2}}{d^{2 N+2}-c^{2 N+2}}} .
$$

We define the $(2 N+1)$-dimensional orthogonal matrix

$$
\mathbf{W}^{\mathrm{P}}=\left(\begin{array}{cc}
\mathbf{Y}^{\mathrm{P}} & \mathbf{0} \\
\mathbf{0} & \mathbf{X}^{\mathrm{P}}
\end{array}\right),
$$

which gives

$$
\left(\mathbf{W}^{\mathrm{P}}\right)^{\mathrm{T}} \mathbf{A}^{\mathrm{P}} \mathbf{W}^{\mathrm{P}}=\left(\begin{array}{cc}
a \mathbf{1}_{N+1} & \left(\mathbf{Y}^{\mathrm{P}}\right)^{\mathrm{T}} \mathbf{B}^{\mathrm{P}} \mathbf{X}^{\mathrm{P}} \\
\left(\mathbf{X}^{\mathrm{P}}\right)^{\mathrm{T}}\left(\mathbf{B}^{\mathrm{P}}\right)^{\mathrm{T}} \mathbf{Y}^{\mathrm{P}} & b \mathbf{1}_{N}
\end{array}\right) .
$$


The off-diagonal blocks in Eq. (2.16) simplify because

$$
\left(\mathbf{Y}^{\mathrm{P}}\right)^{\mathrm{T}} \mathbf{B}^{\mathrm{P}} \mathbf{X}^{\mathrm{P}}=\boldsymbol{\sigma}^{\mathrm{P}}
$$

where $\sigma^{\mathrm{P}}$ is given in Eq. (2.4). It is evident that each block of $\left(\mathbf{W}^{\mathrm{P}}\right)^{\mathrm{T}} \mathbf{A}^{\mathrm{P}} \mathbf{W}^{\mathrm{P}}$ is diagonal, so that it comprises $N$ two-dimensional interacting blocks of the form

$$
\left(\begin{array}{cc}
a & \sigma_{k}^{\mathrm{P}} \\
\sigma_{k}^{\mathrm{P}} & b
\end{array}\right)
$$

and a single one-dimensional block with eigenvalue $a$. The two-dimensional blocks give $2 N$ eigenvalues

$$
E_{\mathrm{k} \pm}^{\mathrm{P}}=\frac{1}{2}(a+b) \pm \frac{1}{2} D_{\mathrm{k}}^{\mathrm{P}} \quad \text { for } \mathrm{k}=1,2, \ldots, N
$$

with discriminant

$$
D_{\mathrm{k}}^{\mathrm{P}}=\sqrt{(a-b)^{2}+4\left(\sigma_{\mathrm{k}}^{\mathrm{P}}\right)^{2}}=\sqrt{(a-b)^{2}+4\left(c^{2}+d^{2}+2 c d \cos \theta_{\mathrm{k}}^{\mathrm{P}}\right)} .
$$

The eigenvectors arising from these two-dimensional blocks can be written as

$$
N_{\mathrm{k} \pm}^{\mathrm{P}}\left(\begin{array}{c}
\sigma_{\mathrm{k}}^{\mathrm{P}} \\
E_{\mathrm{k} \pm}^{\mathrm{P}}-a
\end{array}\right)
$$

where the normalisation constants are

$$
N_{\mathrm{k}+}^{\mathrm{P}}=\sqrt{\frac{1}{D_{\mathrm{k}}^{\mathrm{P}}\left(E_{\mathrm{k}+}^{\mathrm{P}}-a\right)}} \quad \text { and } \quad N_{\mathrm{k}-}^{\mathrm{P}}=\sqrt{\frac{1}{D_{\mathrm{k}}^{\mathrm{P}}\left(a-E_{\mathrm{k}-}^{\mathrm{P}}\right)}} .
$$

We can write the $2 N+1$ eigenvectors of $\mathbf{A}^{\mathrm{P}}$ in the form $\mathbf{c}_{\mathrm{k} \pm}^{\mathrm{P}}$ for $\mathrm{k}=1,2, \ldots, N$, and $\mathbf{c}_{N+1}^{\mathrm{P}}$, the latter arising from the extra null space eigenvector in the singular value decomposition. Using expression (2.12) for $\mathrm{Y}^{\mathrm{P}}$, we obtain expressions for the eigenvector coefficients

$$
\begin{aligned}
c_{2 \mathrm{p}, \mathrm{k} \pm}^{\mathrm{P}} & =N_{\mathrm{k} \pm}^{\mathrm{P}}\left(E_{\mathrm{k} \pm}^{\mathrm{P}}-a\right) X_{\mathrm{pk}}^{\mathrm{P}}, \\
c_{2 \mathrm{p}-1, \mathrm{k} \pm}^{\mathrm{P}} & =N_{\mathrm{k} \pm}^{\mathrm{P}}\left(c X_{\mathrm{pk}}^{\mathrm{P}}+d X_{\mathrm{p}-1, \mathrm{k}}^{\mathrm{P}}\right) .
\end{aligned}
$$

There is, in addition, a single eigenvalue arising from the one-dimensional block, and corresponding to the null-space eigenvector in the $\mathrm{Y}^{\mathrm{P}}$ subspace, that is of the form

$$
E_{N+1}^{\mathrm{P}}=a .
$$

The corresponding eigenvector has coefficients

$$
\begin{aligned}
c_{2 \mathrm{p}, N+1}^{\mathrm{P}} & =0, \\
c_{2 \mathrm{p}-1, N+1}^{\mathrm{P}} & =N_{N+1}^{\mathrm{P}}(-1)^{\mathrm{p}-1} c^{\mathrm{p}-1} d^{N-\mathrm{p}+1} .
\end{aligned}
$$




\subsection{The even path, $P_{2 N}(a, b \mid c, d)$}

The adjacency matrix for the bipartite graph, $P_{2 N}(a, b \mid c, d)$, can be written as

$$
\mathbf{A}^{\mathrm{P}}=\left(\begin{array}{cc}
a \mathbf{1}_{N} & \mathbf{B}^{\mathrm{P}} \\
\left(\mathbf{B}^{\mathrm{P}}\right)^{\mathrm{T}} & b \mathbf{1}_{N}
\end{array}\right),
$$

using the same numbering scheme for vertices as that shown in Fig. 2. The adjacency matrix is identical to Eq. (2.1), but with one row missing, so that $\mathbf{B}^{\mathrm{P}}$ is a square $N \times N$ matrix.

We again use singular decomposition [8, Sections 2.5.3 and 2.5.6] of $\mathbf{B}^{\mathrm{P}}$ as shown in Eq. (2.3), where $\mathbf{X}^{\mathrm{P}}$ and $\mathbf{Y}^{\mathrm{P}}$ are both $N$-dimensional orthogonal matrices. We note that the $N \times N$-dimensional positive definite tridiagonal matrix has the form

$$
\left(\mathbf{B}^{\mathrm{P}}\right)^{\mathrm{T}} \mathbf{B}^{\mathrm{P}}=\left(\begin{array}{cccccc}
c^{2}+d^{2} & c d & 0 & \cdots & 0 & 0 \\
c d & c^{2}+d^{2} & c d & \ddots & & 0 \\
0 & c d & c^{2}+d^{2} & \ddots & \ddots & \vdots \\
\vdots & \ddots & \ddots & \ddots & \ddots & 0 \\
0 & & \ddots & \ddots & c^{2}+d^{2} & c d \\
0 & 0 & \cdots & 0 & c d & c^{2}
\end{array}\right) .
$$

We introduce an Ansatz for the eigenvectors of the matrix in Eq. (2.27) as

$$
X_{\mathrm{pk}}^{\mathrm{P}}=N_{\mathrm{k}}^{\mathrm{P}} \sin \mathrm{p} \theta_{\mathrm{k}}^{\mathrm{P}},
$$

where $N_{\mathrm{k}}^{\mathrm{P}}$ is a normalization factor, and $\theta_{\mathrm{k}}^{\mathrm{P}}$ is an angle yet to be determined. Examining the first row of $\left(\mathbf{B}^{\mathrm{P}}\right)^{\mathrm{T}} \mathbf{B}^{\mathrm{P}} \mathbf{X}^{\mathrm{P}}$, leaving out the normalisation factor, we find

$$
\left(c^{2}+d^{2}\right) \sin \theta_{\mathrm{k}}^{\mathrm{P}}+c d \sin 2 \theta_{\mathrm{k}}^{\mathrm{P}}=\sigma_{\mathrm{k}}^{\mathrm{P}^{2}} \sin \theta_{\mathrm{k}}^{\mathrm{P}},
$$

and expanding $\sin 2 \theta_{\mathrm{k}}^{\mathrm{P}}$, we obtain an expression for the eigenvalue as

$$
\left(\sigma_{\mathrm{k}}^{\mathrm{P}}\right)^{2}=c^{2}+d^{2}+2 c d \cos \theta_{\mathrm{k}}^{\mathrm{P}}
$$

which should be compared with Eq. (2.7). Likewise, for row p,

$$
c d \sin (\mathrm{p}-1) \theta_{\mathrm{k}}^{\mathrm{P}}+\left(c^{2}+d^{2}\right) \sin \mathrm{p} \theta_{\mathrm{k}}^{\mathrm{P}}+c d \sin (\mathrm{p}+1) \theta_{\mathrm{k}}^{\mathrm{P}}=\sigma_{\mathrm{k}}^{\mathrm{P}^{2}} \sin \mathrm{p} \theta_{\mathrm{k}}^{\mathrm{P}} .
$$

Noting that

$$
\sin (\mathrm{p}-1) \theta_{\mathrm{k}}^{\mathrm{P}}+\sin (\mathrm{p}+1) \theta_{\mathrm{k}}^{\mathrm{P}}=2 \sin \mathrm{p} \theta_{\mathrm{k}}^{\mathrm{P}} \cos \theta_{\mathrm{k}}^{\mathrm{P}},
$$

it is easy to see that the eigenvalue equations are satisfied for rows $\mathrm{p}=2$ up to row $N-1$ using the expression Eq. (2.30) for the eigenvalue. However, the $N$ th equation is

$$
c d \sin (N-1) \theta_{\mathrm{k}}^{\mathrm{P}}+c^{2} \sin N \theta_{\mathrm{k}}^{\mathrm{P}}=\sigma_{\mathrm{k}}^{\mathrm{P} 2} \sin N \theta_{\mathrm{k}}^{\mathrm{P}} .
$$

In order to ensure that this equation be satisfied, we would need to add a factor

$$
d^{2} \sin N \theta_{\mathrm{k}}^{\mathrm{P}}+c d \sin (N+1) \theta_{\mathrm{k}}^{\mathrm{P}}
$$


to the left-hand side. Dividing by $\sin \theta_{\mathrm{k}}^{\mathrm{P}}$, it follows that we require a condition on $\theta_{\mathrm{k}}^{\mathrm{P}}$, such that

$$
c U_{N}\left(\cos \theta_{\mathrm{k}}^{\mathrm{P}}\right)+d U_{N-1}\left(\cos \theta_{\mathrm{k}}^{\mathrm{P}}\right)=0 \quad \text { for }-\pi \leq \theta_{\mathrm{k}}^{\mathrm{P}} \leq \pi .
$$

Eq. (2.35) is a polynomial of order $N$ in the variable $\cos \theta_{\mathrm{k}}^{\mathrm{P}}$, and has $N$ solutions that fully determine the spectrum.

The norm of the eigenvectors is obtained from

$$
\begin{aligned}
\sum_{\mathrm{p}=1}^{N}\left(X_{\mathrm{pk}}^{\mathrm{P}}\right)^{2} & =\left(N_{\mathrm{k}}^{\mathrm{P}}\right)^{2} \sum_{\mathrm{p}=1}^{N} \sin ^{2} \mathrm{p} \theta_{\mathrm{k}}^{\mathrm{P}} \\
& =\left(N_{X \mathrm{k}}^{\mathrm{P}}\right)^{2} \frac{1}{4}\left\{2 N+1-\frac{\sin (2 N+1) \theta_{\mathrm{k}}^{\mathrm{P}}}{\sin \theta_{\mathrm{k}}^{\mathrm{P}}}\right\} \\
& =1,
\end{aligned}
$$

where we use a well-known trigonometrical summation [10]. This simplifies to

$$
N_{\mathrm{k}}^{\mathrm{P}}=\frac{2}{\sqrt{2 N+1-U_{2 N}\left(\cos \theta_{\mathrm{k}}^{\mathrm{P}}\right)}},
$$

which has been expressed in terms of the Chebyshev polynomial of the second kind,

$$
U_{2 N}(\cos \theta)=\frac{\sin (2 N+1) \theta}{\sin \theta} .
$$

The $N \times N$-dimensional semi-definite tridiagonal matrix $\mathbf{B}^{\mathrm{P}}\left(\mathbf{B}^{\mathrm{P}}\right)^{\mathrm{T}}$, has eigenvectors $\mathbf{Y}^{\mathrm{P}}$ that can be derived directly from the singular value decomposition as

$$
Y_{\mathrm{pk}}^{\mathrm{P}}=\frac{N_{\mathrm{k}}^{\mathrm{P}}}{\sigma_{\mathrm{k}}^{\mathrm{p}}}\left[c \sin \mathrm{p} \theta_{\mathrm{k}}^{\mathrm{P}}+d \sin (\mathrm{p}-1) \theta_{\mathrm{k}}^{\mathrm{P}}\right] .
$$

The expression for the eigenvalues,

$$
E_{\mathrm{k} \pm}^{\mathrm{P}}=\frac{1}{2}(a+b) \pm \frac{1}{2} D_{\mathrm{k}}^{\mathrm{P}} \quad \text { for } \mathrm{k}=1,2, \ldots, N,
$$

where

$$
D_{\mathrm{k}}^{\mathrm{P}}=\sqrt{(a-b)^{2}+4\left(c^{2}+d^{2}+2 c d \cos \theta_{\mathrm{k}}^{\mathrm{P}}\right)},
$$

is identical to that for the odd path (Eq. (2.19)), apart from the difference in angle $\theta_{\mathrm{k}}^{\mathrm{P}}$. The eigenvector entries are

$$
\begin{aligned}
c_{2 \mathrm{p}, \mathrm{k} \pm}^{\mathrm{P}} & =N_{\mathrm{k} \pm}^{\mathrm{P}}\left(E_{\mathrm{k} \pm}^{\mathrm{P}}-a\right) X_{\mathrm{pk}}^{\mathrm{P}}, \\
c_{2 \mathrm{p}-1, \mathrm{k} \pm}^{\mathrm{P}} & =N_{\mathrm{k} \pm}^{\mathrm{P}}\left(c X_{\mathrm{pk}}^{\mathrm{P}}+d X_{\mathrm{p}-1, \mathrm{k}}^{\mathrm{P}}\right) .
\end{aligned}
$$

We note that the expressions for eigenvalues and eigenvectors of odd and even paths are substantially the same for the pairs $E_{\mathrm{k} \pm}^{\mathrm{P}}$. The odd path has an extra eigenvector arising from the null space of $\mathbf{B}^{\mathrm{P}}\left(\mathbf{B}^{\mathrm{P}}\right)^{\mathrm{T}}$. The expression for the angle $\theta_{\mathrm{k}}^{\mathrm{P}}$, however, is different in the two cases (c.f. Eqs. (2.8) and (2.35)), as are the normalisation factors (c.f. Eqs. (2.10) and (2.37)). The angle $\theta_{\mathrm{k}}^{\mathrm{P}}$ is the sole quantity that cannot be determined in closed form for the even chain. For some values of the edge weights $c, d$, the angle $\theta_{\mathrm{k}}^{\mathrm{P}}$ may be equal to $\pm \pi$. Such cases must be treated separately as the expressions for the norm (2.37) and for the eigenvector entries in Eq. (2.41) vanish, but this is not difficult. 


\section{Structural polynomials of alternating paths}

The characteristic polynomials for alternating paths can be written

$$
\begin{gathered}
s\left(P_{2 N+1}(a, b \mid c, d), E\right)=(E-a) \prod_{\mathrm{k}=1}^{N}\left(E-E_{\mathrm{k}+}^{\mathrm{P}}\right)\left(E-E_{\mathrm{k}-}^{\mathrm{P}}\right), \\
s\left(P_{2 N}(a, b \mid c, d), E\right)=\prod_{\mathrm{k}=1}^{N}\left(E-E_{\mathrm{k}+}^{\mathrm{P}}\right)\left(E-E_{\mathrm{k}-}^{\mathrm{P}}\right) .
\end{gathered}
$$

We can combine these factors in pairs as

$$
\left(E-E_{\mathrm{k}+}^{\mathrm{P}}\right)\left(E-E_{\mathrm{k}-}^{\mathrm{P}}\right)=(E-a)(E-b)-c^{2}-d^{2}-2 c d \cos \theta_{\mathrm{k}}^{\mathrm{P}},
$$

and using the product expression for the Chebyshev function of the second kind,

$$
U_{N}(z)=\prod_{\mathrm{k}=1}^{N}\left(2 z-2 \cos \frac{\mathrm{k} \pi}{N+1}\right),
$$

it can be shown that the 'half-chain' expression for the odd path is

$$
s\left(P_{2 N+1}(a, b \mid c, d), E\right)=(E-a)(c d)^{N} U_{N}(x),
$$

with

$$
x=\frac{(E-a)(E-b)-\left(c^{2}+d^{2}\right)}{2 c d} .
$$

The expression in Eq. (3.3) cannot be used for even paths because of the more complicated formula for the angle, $\theta_{\mathrm{k}}^{\mathrm{P}}$. It has been shown by Gover [9], however, that the even chain has a related 'half-chain' form

$$
s\left(P_{2 N}(a, b \mid c, d), E\right)=d(c d)^{N-1} \widetilde{U}_{N}(x ; c, d),
$$

where

$$
\widetilde{U}_{N}(x ; c, d)=c U_{N}(x)+d U_{N-1}(x) .
$$

We can derive an expression in terms of Chebyshev polynomials for the full chain by using the standard formula

$$
U_{2 N+1}(z)=2 z U_{N}\left(2 z^{2}-1\right)
$$

which, when applied to Eqs. (3.4) and (3.6) gives

$$
\begin{aligned}
s\left(P_{2 N+1}(a, b \mid c, d), E\right) & =\frac{(c d)^{N}(E-a)}{2 y} U_{2 N+1}(y), \\
s\left(P_{2 N}(a, b \mid c, d), E\right) & =\frac{d(c d)^{N-1}}{2 y}\left(c U_{2 N+1}(y)+d U_{2 N-1}(y)\right),
\end{aligned}
$$

where, comparing Eqs. (3.4), (3.6) and (3.8) gives

$$
y=\sqrt{\frac{(E-a)(E-b)-(c-d)^{2}}{4 c d}} .
$$


In the case of a non-alternating linear chain, with $a=b$ and $c=d$, Eqs. (3.9) simplify to

$$
\begin{aligned}
s\left(P_{2 N+1}(a, a \mid c, c), E\right) & =c^{2 N+1} U_{2 N+1}\left(\frac{E-a}{2 c}\right), \\
s\left(P_{2 N}(a, a \mid c, c), E\right) & =\frac{c^{2 N+1}}{E-a}\left(U_{2 N+1}\left(\frac{E-a}{2 c}\right)+U_{2 N-1}\left(\frac{E-a}{2 c}\right)\right) \\
& =c^{2 N} U_{2 N}\left(\frac{E-a}{2 c}\right),
\end{aligned}
$$

where we have used the Chebyshev recursion relations in the last step.

Closed-form expressions for the other structural polynomials can be derived using the explicit inverse for 2-Toeplitz matrices derived by da Fonseca and Petronilho [2], and using Eqs. (1.1) and (1.2). Making the necessary translation of notation, we find that for paths of order $2 N+1$ and assuming that $\mathrm{p} \leq \mathrm{q}$,

$$
\begin{aligned}
\jmath_{2 \mathrm{p}, 2 \mathrm{q}}\left(P_{2 N+1}(a, b \mid c, d), E\right) & =(c d)^{N-1}(E-a)^{2} U_{\mathrm{p}-1}(x) U_{N-\mathrm{q}}(x), \\
\jmath_{2 \mathrm{p}-1,2 \mathrm{q}-1}\left(P_{2 N+1}(a, b \mid c, d), E\right) & =(c d)^{N-1} \widetilde{U}_{\mathrm{p}-1}(x ; c, d) \widetilde{U}_{N+1-\mathrm{q}}(x ; d, c), \\
\jmath_{2 \mathrm{p}, 2 \mathrm{q}+1}\left(P_{2 N+1}(a, b \mid c, d), E\right) & =(c d)^{N-1}(E-a) U_{\mathrm{p}-1}(x) \widetilde{U}_{N-\mathrm{q}}(x ; d, c), \\
\jmath_{2 \mathrm{p}-1,2 \mathrm{q}}\left(P_{2 N+1}(a, b \mid c, d), E\right) & =(c d)^{N-1}(E-a) \widetilde{U}_{\mathrm{p}-1}(x ; c, d) U_{N-\mathrm{q}}(x) .
\end{aligned}
$$

The expressions for $2 N$-vertex paths, again assuming that $\mathrm{p} \leq \mathrm{q}$, are

$$
\begin{aligned}
\jmath_{2 \mathrm{p}, 2 \mathrm{q}}\left(P_{2 N}(a, b \mid c, d), E\right) & =d(c d)^{N-2}(E-a) U_{\mathrm{p}-1}(x) \widetilde{U}_{N-\mathrm{q}}(x ; c, d), \\
\jmath_{2 \mathrm{p}-1,2 \mathrm{q}-1}\left(P_{2 N}(a, b \mid c, d), E\right) & =d(c d)^{N-2}(E-b) \widetilde{U}_{\mathrm{p}-1}(x ; c, d) U_{N-\mathrm{q}}(x), \\
\jmath_{2 \mathrm{p}, 2 \mathrm{q}+1}\left(P_{2 N}(a, b \mid c, d), E\right) & =d(c d)^{N-2}(E-a)(E-b) U_{\mathrm{p}-1}(x) U_{N-\mathrm{q}-1}(x), \\
\jmath_{2 \mathrm{p}-1,2 \mathrm{q}}\left(P_{2 N}(a, b \mid c, d), E\right) & =d(c d)^{N-2} \widetilde{U}_{\mathrm{p}-1}(x ; c, d) \widetilde{U}_{N-\mathrm{q}}(x ; c, d) .
\end{aligned}
$$

For cases where $q<p$, one needs to swap indices p and $q$ in Eqs. (3.12) and (3.13).

\section{Alternating ladders $L_{2 M}(a, b \mid c, d)$}

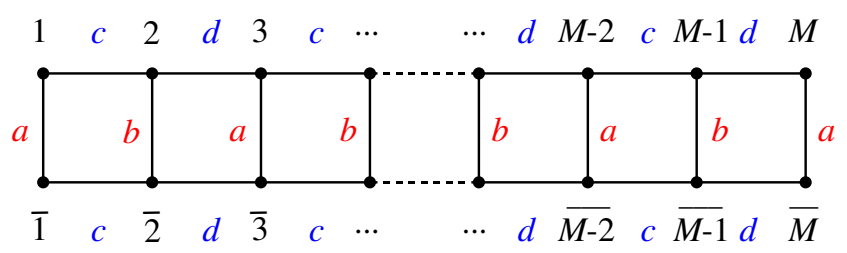

Figure 3: A linear ladder, $L_{2 M}(a, b \mid c, d)$, with $2 M$ vertices and alternating rung $(a, b)$ and riser weights $(c, d)$.

The $2 M$-vertex linear ladder, $L_{2 M}(a, b \mid c, d)$, has two alternating $M$-vertex paths $(1,2, \ldots, M)$ and $(\overline{1}, \overline{2}, \ldots, \bar{M})$ joined by rungs between like-numbered vertices, $(\mathrm{p}, \overline{\mathrm{p}})$, with alternating weights as displayed in Fig. 3. The quantities $(a, b)$ are, in this case, rung weights, and $(c, d)$ are edge weights for the two riser chains. 
The ladder has an involution involving simultaneous exchange of all vertices attached to the ends of the rungs. This involution can be exhibited by arranging the adjacency matrix so that the vertices are first put into $2 \times 2$ blocks with vertices 1 to $M$ of the first path followed by vertices $\overline{1}$ to $\bar{M}$ of the lower path. Next, odd vertices $1,3, \ldots$ are placed in a block together, and then the even vertices, $2,4, \ldots$ The same procedure is adopted for the lower path. We now have a $4 \times 4$ blocked adjacency matrix in the form:

$$
\left(\begin{array}{cccc}
\mathbf{0} & \mathbf{B}^{\mathrm{P}} & a \mathbf{1} & \mathbf{0} \\
\left(\mathbf{B}^{\mathrm{P}}\right)^{\mathrm{T}} & \mathbf{0} & \mathbf{0} & b \mathbf{1} \\
a \mathbf{1} & \mathbf{0} & \mathbf{0} & \mathbf{B}^{\mathrm{P}} \\
\mathbf{0} & b \mathbf{1} & \left(\mathbf{B}^{\mathrm{P}}\right)^{\mathrm{T}} & \mathbf{0}
\end{array}\right)
$$

where $\mathbf{B}^{\mathrm{P}}$ is the same matrix as in Eq. (2.2). This matrix can be block-diagonalised by an orthogonal transformation of the form

$$
\left(\begin{array}{rr}
\frac{1}{\sqrt{2}} \mathbf{1}_{M} & \frac{1}{\sqrt{2}} \mathbf{1}_{M} \\
\frac{1}{\sqrt{2}} \mathbf{1}_{M} & -\frac{1}{\sqrt{2}} \mathbf{1}_{M}
\end{array}\right)
$$

where the blocks are over all $M$ vertices of top and bottom paths. This transformation leads to the adjacency matrix

$$
\left(\begin{array}{cccc}
a \mathbf{1} & \mathbf{B}^{\mathrm{P}} & \mathbf{0} & \mathbf{0} \\
\left(\mathbf{B}^{\mathrm{P}}\right)^{\mathrm{T}} & b \mathbf{1} & \mathbf{0} & \mathbf{0} \\
\mathbf{0} & \mathbf{0} & -a \mathbf{1} & \mathbf{B}^{\mathrm{P}} \\
\mathbf{0} & \mathbf{0} & \left(\mathbf{B}^{\mathrm{P}}\right)^{\mathrm{T}} & -b \mathbf{1}
\end{array}\right)=\left(\begin{array}{cc}
\mathbf{A}^{\mathrm{P}} & \mathbf{0} \\
\mathbf{0} & \mathbf{A}^{\overline{\mathrm{P}}}
\end{array}\right),
$$

in which $\mathbf{A}^{\mathrm{P}}$ is the adjacency matrix of $P_{M}(a, b \mid c, d)$ as in Eq. (2.1), and $\mathbf{A}^{\overline{\mathrm{P}}}$ is the adjacency matrix of $P_{M}(-a,-b \mid c, d)$, the path with vertex weights of opposite sign. It follows that we can use the results of Section 2 to derive expressions for all relevant quantities. The eigenvalues for $L_{2 M}$ are thus

$$
\begin{aligned}
E_{\mu}^{\mathrm{L}} & =E_{\mu}^{\mathrm{P}}, \\
E_{\bar{\mu}}^{(\mathrm{L})} & =E_{\mu}^{\overline{\mathrm{P}}} \quad \text { for } \mu=1,2, \ldots, M,
\end{aligned}
$$

where $E_{\mu}^{\mathrm{P}}$ is an index ranging over the eigenvalues of the path $P_{M}(a, b \mid c, d)$ as given by Eq. (2.39), or (2.19) and (2.24), depending on whether $M$ is even or odd. The eigenfunctions can be written using the vector $\mathbf{c}^{\mathrm{L}}$ using Eq. (2.23) as

$$
\begin{array}{ll}
c_{\mathrm{p} \mu}^{\mathrm{L}}=\frac{c_{\mathrm{p} \mu}^{\mathrm{P}}}{\sqrt{2}}, & c_{\overline{\mathrm{p}} \mu}^{\mathrm{L}}=\frac{c_{\overline{\mathrm{p}} \mu}^{\mathrm{P}}}{\sqrt{2}}, \\
c_{\mathrm{p} \bar{\mu}}^{\mathrm{L}}=\frac{c_{\mathrm{p} \mu}^{\overline{\mathrm{P}}}}{\sqrt{2}}, & c_{\overline{\mathrm{p}} \bar{\mu}}^{\mathrm{L}}=-\frac{c_{\overline{\mathrm{p}} \mu}^{\overline{\mathrm{P}}}}{\sqrt{2}}
\end{array}
$$

where the index, $\mu=1,2, \ldots, M$, labels the eigenvectors in each of the symmetric and antisymmetric blocks, labelled $\mu$ and $\bar{\mu}$, respectively. 


\section{Structural polynomials of alternating ladders}

The characteristic polynomial, $s^{L_{M}}(E)$, can be derived directly from the expressions in Section 3 to give

$$
\begin{aligned}
s^{L_{4 N}} & =d^{2}(c d)^{2 N-2} \widetilde{U}_{N}(x ; c, d) \widetilde{U}_{N}(\bar{x} ; c, d), \\
s^{L_{4 N+2}} & =\left(E^{2}-a^{2}\right)(c d)^{2 N} U_{N}(x) U_{N}(\bar{x}),
\end{aligned}
$$

where we use $x$ defined in Eq. (3.5) along with the antisymmetric analogue

$$
\bar{x}=\frac{(E+a)(E+b)-c^{2}-d^{2}}{2 c d} .
$$

We can also use the results of Section 3 to write

$$
\begin{aligned}
s^{L_{4 N}} & =\frac{d^{2}}{4 y \bar{y}}(c d)^{2 N-2}\left(c U_{2 N+1}(y)+d U_{2 N-1}(y)\right)\left(c U_{2 N+1}(\bar{y})+d U_{2 N-1}(\bar{y})\right), \\
s^{L_{4 N+2}} & =\frac{\left(E^{2}-a^{2}\right)}{4 y \bar{y}}(c d)^{2 N} U_{2 N+1}(y) U_{2 N+1}(\bar{y}),
\end{aligned}
$$

in which we use

$$
\bar{y}=\sqrt{\frac{(E+a)(E+b)-(c-d)^{2}}{4 c d}},
$$

along with the definition of $y$ in Eq. (3.10). The characteristic polynomials for the ladder, therefore, are written in Eqs. (5.1) and (5.3) as products of characteristic polynomials for the part systems $P_{M}(a, b \mid c, d)$ and $P_{M}(-a,-b \mid c, d)$.

We can easily deduce the forms of the $\jmath_{\mathrm{pq}}$ structural polynomials, since, using the spectral expansion of the structural polynomials in Eq. (1.5),

$$
J_{\mathrm{pq}}^{L_{M}}=\left(E \mathbf{1}-\mathbf{A}^{\mathrm{L}}\right)_{\mathrm{pq}}^{-1} s^{\mathrm{L}}(E)=\frac{1}{2} \sum_{\mu=1}^{M}\left\{\frac{c_{\mathrm{p} \mu}^{\mathrm{P}} c_{\mathrm{q} \mu}^{\mathrm{P}}}{E-E_{\mu}^{\mathrm{P}}}+\frac{c_{\mathrm{p} \mu}^{\overline{\mathrm{P}}} c_{\mathrm{q} \mu}^{\overline{\mathrm{P}}}}{E-E_{\mu}^{\overline{\mathrm{P}}}}\right\} s^{\mathrm{L}}(E) .
$$

It follows immediately that,

$$
J_{\mathrm{pq}}^{L_{M}}=\frac{1}{2}\left\{\int_{\mathrm{pq}}^{\mathrm{P}}(E) s^{\overline{\mathrm{P}}}(E)+s^{\mathrm{P}}(E) j_{\mathrm{pq}}^{\overline{\mathrm{P}}}(E)\right\}
$$

which also exhibits a simple structure in terms of $P_{M}(a, b \mid c, d)$ and $P_{M}(-a,-b \mid c, d)$.

The structural polynomials for $L_{4 N+2}(a, b \mid c, d)$, expressed in terms of the half-ladder, 
where both vertices are on the same backbone path, and assuming $\mathrm{p} \leq \mathrm{q}$, are

$$
\begin{aligned}
& j_{2 \mathrm{p}, 2 \mathrm{q}}^{L_{4 N+2}}=\frac{E^{2}-a^{2}}{2}(c d)^{2 N-1}\left\{(E-a) U_{\mathrm{p}-1}(x) U_{N-\mathrm{q}}(x) U_{N}(\bar{x})\right. \\
&+\left.(E+a) U_{N}(x) U_{\mathrm{p}-1}(\bar{x}) U_{N-\mathrm{q}}(\bar{x})\right\}, \\
& j_{2 \mathrm{p}-1,2 \mathrm{q}-1}^{L_{4 N+2}}=\frac{1}{2}(c d)^{2 N-1}\left\{(E+a) \widetilde{U}_{\mathrm{p}-1}(x ; c, d) \widetilde{U}_{N+1-\mathrm{q}}(x ; d, c) U_{N}(\bar{x})\right.\left.+(E-a) U_{N}(x) \widetilde{U}_{\mathrm{p}-1}(\bar{x} ; c, d) \widetilde{U}_{N+1-\mathrm{q}}(\bar{x} ; d, c)\right\}, \\
&\left.+U_{N}(x) U_{\mathrm{p}-1}(\bar{x}) \widetilde{U}_{N-\mathrm{q}}(\bar{x} ; d, c)\right\}, \\
& J_{2 \mathrm{p}, 2 \mathrm{q}+1}^{L_{4 N+2}}=\frac{E^{2}-a^{2}}{2}(c d)^{2 N-1}\left\{U_{\mathrm{p}-1}(x) \widetilde{U}_{N-\mathrm{q}}(x ; d, c) U_{N}(\bar{x})\right. \\
& J_{2 \mathrm{p}-1,2 \mathrm{q}}=\frac{E^{2}-a^{2}}{2}(c d)^{2 N-1}\left\{\widetilde{U}_{\mathrm{p}-1}(x ; c, d) U_{N-\mathrm{q}}(x) U_{N}(\bar{x})\right. \\
&+\left.U_{N}(x) \widetilde{U}_{\mathrm{p}-1}(\bar{x} ; c, d) U_{N-\mathrm{q}}(\bar{x})\right\} .
\end{aligned}
$$

If $\mathrm{p}>\mathrm{q}$, then the indices $\mathrm{p}$ and $\mathrm{q}$ are swapped on the right-hand side of Eq. (5.7). For vertices on different backbone chains, and assuming again $\mathrm{p} \leq \mathrm{q}$,

$$
\begin{aligned}
& J_{2 \mathrm{p}, 2 \overline{\mathrm{q}}}^{L_{4 N+2}}=\frac{E^{2}-a^{2}}{2}(c d)^{2 N-1}\left\{(E-a) U_{\mathrm{p}-1}(x) U_{n-\mathrm{q}}(x) U_{N}(\bar{x})\right. \\
& \left.-(E+a) U_{N}(x) U_{\mathrm{p}-1}(\bar{x}) U_{N-\mathrm{q}}(\bar{x})\right\}, \\
& J_{2 \mathrm{p}-1,2 \overline{\mathrm{q}}-1}^{L_{4 N+2}}=\frac{1}{2}(c d)^{2 N-1}\left\{(E+a) \widetilde{U}_{\mathrm{p}-1}(x ; c, d) \widetilde{U}_{N+1-\mathrm{q}}(x ; d, c) U_{N}(\bar{x})\right. \\
& \left.-(E-a) U_{N}(x) \widetilde{U}_{\mathrm{p}-1}(\bar{x} ; c, d) \widetilde{U}_{N+1-\mathrm{q}}(\bar{x} ; d, c)\right\} \text {, } \\
& \jmath_{2 \mathrm{p}, 2 \overline{\mathrm{q}}+1}^{L_{4 N+2}}=\frac{E^{2}-a^{2}}{2}(c d)^{2 N-1}\left\{U_{\mathrm{p}-1}(x) \widetilde{U}_{N-\mathrm{q}}(x ; d, c) U_{N}(\bar{x})\right. \\
& \left.-U_{N}(x) U_{\mathrm{p}-1}(\bar{x}) \widetilde{U}_{N-\mathrm{q}}(\bar{x} ; d, c)\right\} \text {, } \\
& \jmath_{2 \mathrm{p}-1,2 \overline{\mathrm{q}}}^{L_{4 N+2}}=\frac{E^{2}-a^{2}}{2}(c d)^{2 N-1}\left\{\widetilde{U}_{\mathrm{p}-1}(x ; c, d) U_{N-\mathrm{q}}(x) U_{N}(\bar{x})\right. \\
& \left.-U_{N}(x) \widetilde{U}_{\mathrm{p}-1}(\bar{x} ; c, d) U_{N-\mathrm{q}}(\bar{x})\right\} \text {. }
\end{aligned}
$$

If $\mathrm{p}>\mathrm{q}$, then the indices $\mathrm{p}$ and $\mathrm{q}$ are swapped on the right-hand side of Eq. (5.8). Comparing Eqs. (5.7) and (5.8), we observe a sign change in the expressions that arises from the sign patterns of the antisymmetric functions in Eq. (4.5).

The structural polynomials, expressed in terms of the half-ladder for $L_{4 N}(a, b \mid c, d)$, 
where both vertices are on the same backbone path and $\mathrm{p} \leq \mathrm{q}$, are

$$
\begin{aligned}
& j_{2 \mathrm{p}, 2 \mathrm{q}}^{L_{4 N}}=\frac{1}{2} d^{2}(c d)^{2 N-3}\left\{(E-a) U_{\mathrm{p}-1}(x) \widetilde{U}_{N-\mathrm{q}}(x ; c, d) \widetilde{U}_{N}(\bar{x} ; c, d)\right. \\
&+\left.(E+a) \widetilde{U}_{N}(x ; c, d) U_{\mathrm{p}-1}(\bar{x}) \widetilde{U}_{N-\mathrm{q}}(\bar{x} ; c, d)\right\}, \\
& J_{2 \mathrm{p}-1,2 \mathrm{q}-1}^{L_{4 N}}=\frac{1}{2} d^{2}(c d)^{2 N-3}\left\{(E-b) \widetilde{U}_{\mathrm{p}-1}(x ; c, d) U_{N-\mathrm{q}}(x) \widetilde{U}_{N}(\bar{x} ; c, d)\right. \\
&\left.+(E+b) \widetilde{U}_{N}(x ; c, d) \widetilde{U}_{\mathrm{p}-1}(\bar{x} ; c, d) U_{N-\mathrm{q}}(\bar{x})\right\}, \\
& J_{2 \mathrm{p}, 2 \mathrm{q}+1}^{L_{4 N}}=\frac{1}{2} d^{2}(c d)^{2 N-3}\left\{(E-a)(E-b) U_{\mathrm{p}-1}(x) U_{N-\mathrm{q}-1}(x) \widetilde{U}_{N}(\bar{x} ; c, d)\right. \\
&\left.+(E+a)(E+b) \widetilde{U}_{N}(x ; c, d) U_{\mathrm{p}-1}(\bar{x}) U_{N-\mathrm{q}-1}(\bar{x})\right\}, \\
& J_{2 \mathrm{p} s-1,2 \mathrm{q}}^{L_{4 N}}=\frac{1}{2} d^{2}(c d)^{2 N-3}\left\{\widetilde{U}_{\mathrm{p}-1}(x ; c, d) \widetilde{U}_{N-\mathrm{q}}(x ; c, d) \widetilde{U}_{N}(\bar{x} ; c, d)\right. \\
&\left.+\widetilde{U}_{N}(x ; c, d) \widetilde{U}_{\mathrm{p}-1}(\bar{x} ; c, d) \widetilde{U}_{N-\mathrm{q}}(\bar{x} ; c, d)\right\} .
\end{aligned}
$$

If $p>q, p$ and $q$ are swapped on the RHS of Eq. (5.9). For vertices on different backbone paths and $\mathrm{p} \leq \mathrm{q}$,

$$
\begin{aligned}
& \jmath_{2 \mathrm{p}, 2 \mathrm{q}}^{L_{4 N}}=\frac{1}{2} d^{2}(c d)^{2 N-3}\left\{(E-a) U_{\mathrm{p}-1}(x) \widetilde{U}_{N-\mathrm{q}}(x ; c, d) \widetilde{U}_{N}(\bar{x} ; c, d)\right. \\
& \left.-(E+a) \widetilde{U}_{N}(x ; c, d) U_{\mathrm{p}-1}(\bar{x}) \widetilde{U}_{N-\mathrm{q}}(\bar{x} ; c, d)\right\}, \\
& J_{2 \mathrm{p}-1,2 \mathrm{q}-1}^{L_{4 N}}=\frac{1}{2} d^{2}(c d)^{2 N-3}\left\{(E-b) \widetilde{U}_{\mathrm{p}-1}(x ; c, d) U_{N-\mathrm{q}}(x) \widetilde{U}_{N}(\bar{x} ; c, d)\right. \\
& \left.-(E+b) \widetilde{U}_{N}(x ; c, d) \widetilde{U}_{\mathrm{p}-1}(\bar{x} ; c, d) U_{N-\mathrm{q}}(\bar{x})\right\}, \\
& J_{2 \mathrm{p}, 2 \mathrm{q}+1}^{L_{4 N}}=\frac{1}{2} d^{2}(c d)^{2 N-3}\left\{(E-a)(E-b) U_{\mathrm{p}-1}(x) U_{N-\mathrm{q}-1}(x) \widetilde{U}_{N}(\bar{x} ; c, d)\right. \\
& \left.-(E+a)(E+b) \widetilde{U}_{N}(x ; c, d) U_{\mathrm{p}-1}(\bar{x}) U_{N-\mathrm{q}-1}(\bar{x})\right\}, \\
& j_{2 \mathrm{p} s-1,2 \mathrm{q}}^{L_{4 N}}=\frac{1}{2} d^{2}(c d)^{2 N-3}\left\{\widetilde{U}_{\mathrm{p}-1}(x ; c, d) \widetilde{U}_{N-\mathrm{q}}(x ; c, d) \widetilde{U}_{N}(\bar{x} ; c, d)\right. \\
& \left.-\widetilde{U}_{N}(x ; c, d) \widetilde{U}_{\mathrm{p}-1}(\bar{x} ; c, d) \widetilde{U}_{N-\mathrm{q}}(\bar{x} ; c, d)\right\} \text {. }
\end{aligned}
$$

which exhibit the same sign change as in Eq. (5.8) for $L_{4 N+2}$ ladder. If p $>$ q, then $\mathrm{p}$ and q are swapped on the RHS of Eq. (5.10).

\section{Alternating cycles}

We restrict our attention to even cycles with alternating weights. We consider separately the standard cycle, $C_{2 N}(a, b \mid c, d)$, and the flipped cycle, $C_{2 N}^{f}(a, b \mid c, d)$.

\subsection{Alternating cycles, $C_{2 N}(a, b \mid c, d)$}

The $2 N$-vertex cycle, $C_{2 N}(a, b \mid c, d)$, has alternating weights as displayed in Fig. 4 . The quantities $(a, b)$ are in this case vertex weights, and $(c, d)$ are edge weights. It is convenient 


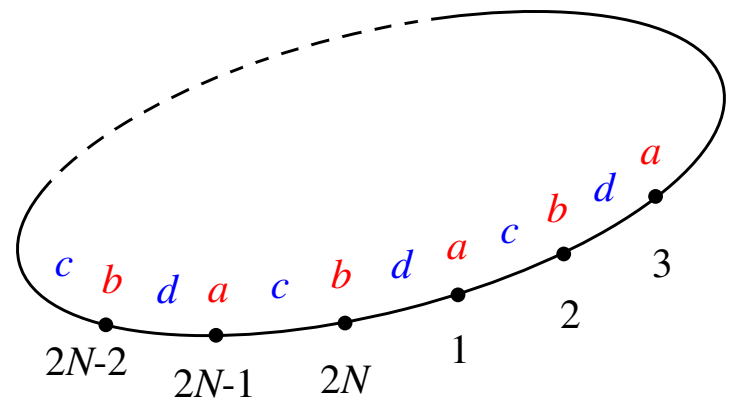

Figure 4: A ring, $C_{2 N}(a, b \mid c, d)$, with $2 N$ vertices and alternating vertex weights $(a, b)$ and edge weights $(c, d)$.

to write the adjacency matrix for this bipartite graph as

$$
\mathbf{A}^{\mathrm{C}}=\left(\begin{array}{cc}
a \mathbf{1}_{N} & \mathbf{B}^{\mathrm{C}} \\
\left(\mathbf{B}^{\mathrm{C}}\right)^{\mathrm{T}} & b \mathbf{1}_{N}
\end{array}\right)
$$

where we have placed the $N$ odd-numbered vertices in the first block, and the $N$ evennumbered vertices in the second. The $N \times N$ matrix $\mathbf{B}^{\mathrm{C}}$ is

$$
\mathbf{B}^{\mathrm{C}}=\left(\begin{array}{ccccc}
c & 0 & \cdots & 0 & d \\
d & c & \ddots & & 0 \\
0 & \ddots & \ddots & \ddots & \vdots \\
\vdots & \ddots & \ddots & \ddots & 0 \\
0 & \cdots & 0 & d & c
\end{array}\right) .
$$

$\mathbf{B}^{\mathrm{C}}$ is the adjacency matrix of a directed, weighted $N$-cycle. It is easy to show that

$$
\mathbf{B}^{\mathrm{C}} \mathbf{X}^{\mathrm{C}}=\mathbf{X}^{\mathrm{C}} \mathbf{\Omega}^{\mathrm{C}} \text {, }
$$

where

$$
\boldsymbol{\Omega}_{\mathrm{kk}^{\prime}}^{\mathrm{C}}=\delta_{\mathrm{kk}^{\prime}}\left(c+d\left(\omega^{\mathrm{C}}\right)^{-\mathrm{k}}\right),
$$

with

$$
\omega^{\mathrm{C}}=\exp \left(\frac{2 \pi \imath}{N}\right)
$$

The kth eigenvector has entries

$$
X_{\mathrm{pk}}^{\mathrm{C}}=\frac{1}{\sqrt{N}} \exp \left(\frac{2 \pi \imath \mathrm{kp}}{N}\right) \quad \text { for } \mathrm{p}, \mathrm{k}=1,2, \ldots, N .
$$

We use Eq. (6.6) to define the $2 N$-dimensional unitary matrix

$$
\mathbf{W}=\left(\begin{array}{cc}
\mathbf{X}^{\mathrm{C}} & \mathbf{0} \\
\mathbf{0} & \mathbf{X}^{\mathrm{C}}
\end{array}\right)
$$


so that

$$
\mathbf{W}^{\dagger} \mathbf{A}^{\mathrm{C}} \mathbf{W}=\left(\begin{array}{cc}
a \mathbf{1}_{N} & \mathbf{X}^{\mathrm{C} \dagger} \mathbf{B}^{\mathrm{C}} \mathbf{X}^{\mathrm{C}} \\
\mathbf{X}^{\mathrm{C} \dagger}\left(\mathbf{B}^{\mathrm{C}}\right)^{\mathrm{T}} \mathbf{X}^{\mathrm{C}} & b \mathbf{1}_{N}
\end{array}\right)
$$

where the $\dagger$ sign denotes the Hermitian conjugate. This transformation achieves a block diagonalization comprising $N$ two-dimensional blocks of the form

$$
\left(\begin{array}{cc}
a & c+d\left(\omega^{\mathrm{C}}\right)^{-\mathrm{k}} \\
c+d\left(\omega^{\mathrm{C}}\right)^{\mathrm{k}} & b
\end{array}\right)
$$

each with eigenvalues

$$
E_{\mathrm{k} \pm}^{\mathrm{C}}=\frac{1}{2}(a+b) \pm \frac{1}{2} D_{\mathrm{k}}^{\mathrm{C}} \quad \text { for } \mathrm{k}=1, \ldots, N,
$$

where the discriminant is

$$
D_{\mathrm{k}}^{\mathrm{C}}=\sqrt{(a-b)^{2}+4\left(c^{2}+d^{2}+2 c d \cos \left(\frac{2 \pi \mathrm{k}}{N}\right)\right)} .
$$

The eigenvectors can be written as entries in the vector $\mathbf{c}^{\mathrm{C}}$ :

$$
\begin{aligned}
c_{2 \mathrm{p}-1, \mathrm{k} \pm}^{\mathrm{C}} & =N_{\mathrm{k} \pm}^{\mathrm{C}} X_{\mathrm{pk}}^{\mathrm{C}}\left(E_{\mathrm{k} \pm}^{\mathrm{C}}-b\right), \\
c_{2 \mathrm{p}, \mathrm{k} \pm}^{\mathrm{C}} & =N_{\mathrm{k} \pm}^{\mathrm{C}}\left(c X_{\mathrm{pk}}^{\mathrm{C}}+d X_{\mathrm{p}+1, \mathrm{k}}^{\mathrm{C}}\right) .
\end{aligned}
$$

The normalisation constants are

$$
N_{\mathrm{k}+}^{\mathrm{C}}=\sqrt{\frac{1}{D_{\mathrm{k}}^{\mathrm{C}}\left(E_{\mathrm{k}+}^{\mathrm{C}}-b\right)}} \quad \text { and } \quad N_{\mathrm{k}-}^{\mathrm{C}}=\sqrt{\frac{1}{D_{\mathrm{k}}^{\mathrm{C}}\left(b-E_{\mathrm{k}-}^{\mathrm{C}}\right)}} .
$$

\subsection{Flipped alternating cycles, $C_{2 N}^{f}(a, b \mid c, d)$}

The $2 N$-vertex cycle, $C_{2 N}^{f}(a, b \mid c, d)$, has alternating weights as displayed in Fig. 4, except that a single weight has a changed sign; without loss of generality, we shall flip the $(1,2 N)$ edge. It is convenient to write the adjacency matrix as

$$
\mathbf{A}^{\mathrm{C}^{f}}=\left(\begin{array}{cc}
a \mathbf{1}_{N} & \mathbf{B}^{\mathrm{C}^{f}} \\
\left(\mathbf{B}^{\mathrm{C}^{f}}\right)^{\mathrm{T}} & b \mathbf{1}_{N}
\end{array}\right)
$$

in the same manner as in Section 6. The $N$-dimensional matrix $\mathbf{B}^{\mathrm{C}^{f}}$ is hence defined by

$$
\mathbf{B}^{C^{f}}=\left(\begin{array}{ccccc}
c & 0 & \cdots & 0 & -d \\
d & c & \ddots & & 0 \\
0 & \ddots & \ddots & \ddots & \vdots \\
\vdots & \ddots & \ddots & \ddots & 0 \\
0 & & \ddots & d & c \\
0 & 0 & \cdots & 0 & d
\end{array}\right) .
$$


Proceeding as before,

$$
\mathbf{B}^{\mathrm{C}^{f}} \mathbf{X}^{\mathrm{C}^{f}}=\mathbf{X}^{\mathrm{C}^{f}} \mathbf{\Omega}^{\mathrm{C}^{f}}
$$

where

$$
\Omega_{\mathrm{kk}^{\prime}}^{\mathrm{C}^{f}}=\delta \mathrm{kk}^{\prime}\left(c+d\left(\omega^{\mathrm{C}^{f}}\right)^{-(2 \mathrm{k}-1)}\right),
$$

with

$$
\omega^{\mathrm{C}^{f}}=\exp \left(\frac{\imath \pi}{N}\right)
$$

and the eigenfunctions are

$$
X_{\mathrm{pk}}^{\mathrm{C}^{f}}=\frac{1}{\sqrt{N}} \exp \left\{\frac{\imath \pi(2 \mathrm{k}-1) \mathrm{p}}{N}\right\} \quad \text { for } \mathrm{p}, \mathrm{k}=1,2, \ldots, N .
$$

The derivation proceeds exactly as for the simple cycle. The eigenvalues are

$$
E_{\mathrm{k} \pm}^{\mathrm{C}^{f}}=\frac{1}{2}(a+b) \pm \frac{1}{2} D_{\mathrm{k}}^{\mathrm{C}^{f}} \quad \text { for } \mathrm{k}=1, \ldots, N
$$

and the discriminant is

$$
D_{\mathrm{k}}^{\mathrm{C}^{f}}=\sqrt{(a-b)^{2}+4\left(c^{2}+d^{2}+2 c d \cos \left(\frac{\pi(2 \mathrm{k}-1)}{N}\right)\right)} .
$$

The eigenvectors are written as entries in the vector $\mathbf{c}^{\mathrm{C} f}$ as

$$
\begin{aligned}
c_{2 \mathrm{p}-1, \mathrm{k} \pm}^{\mathrm{C}^{f}} & =N_{\mathrm{k} \pm}^{\mathrm{C}^{f}} X_{\mathrm{pk}}^{\mathrm{C}^{f}}\left(E_{\mathrm{k} \pm}^{\mathrm{C}^{f}}-b\right), \\
c_{2 \mathrm{p}, \mathrm{k} \pm}^{\mathrm{C}^{f}} & =N_{\mathrm{k} \pm}^{\mathrm{C}^{f}}\left(c X_{\mathrm{pk}}^{\mathrm{C}^{f}}+d X_{\mathrm{p}+1, \mathrm{k}}^{\mathrm{C}^{f}}\right),
\end{aligned}
$$

which is the analogue of Eq. (6.12), and the normalisation constants are

$$
N_{\mathrm{k}+}^{\mathrm{C}^{f}}=\sqrt{\frac{1}{D_{\mathrm{k}}^{\mathrm{C}^{f}}\left(E_{\mathrm{k}+}^{\mathrm{C}^{f}}-a\right)}} \quad \text { and } \quad N_{\mathrm{k}-}^{\mathrm{C}^{f}}=\sqrt{\frac{1}{D_{\mathrm{k}}^{\mathrm{C}^{f}}\left(a-E_{\mathrm{k}-}^{\mathrm{C}^{f}}\right)}} .
$$

\section{Structural polynomials of alternating cycles}

We derive expressions for the structural polynomials of even cycles and flipped cycles in this section. The characteristic polynomial, $s^{C_{2 N}}(E)$, for the graph $C_{2 N}(a, b \mid c, d)$ is

$$
\begin{aligned}
s^{C_{2 N}} & =\prod_{\mathrm{k}=1}^{N}\left(E-E_{\mathrm{k}+}^{\mathrm{C}}\right)\left(E-E_{\mathrm{k}-}^{\mathrm{C}}\right) \\
& =\prod_{\mathrm{k}=1}^{N}\left((E-a)(E-b)-c^{2}-d^{2}-2 c d \cos \frac{2 \pi \mathrm{k}}{N}\right) .
\end{aligned}
$$

Expressing the cosine in terms of the half-angle, we find that

$$
\begin{aligned}
s^{C_{2 N}} & =(4 c d)^{N} \prod_{\mathrm{k}=1}^{N}\left(\frac{(E-a)(E-b)-(c-d)^{2}}{4 c d}-\cos ^{2} \frac{2 \pi \mathrm{k}}{2 N}\right) \\
& =(4 c d)^{N} \prod_{\mathrm{k}=1}^{2 N}\left\{y-\cos \frac{2 \pi \mathrm{k}}{2 N}\right\}
\end{aligned}
$$


where $y$ is defined in Eq. (3.10). We use the well-known relation

$$
2 T_{N}(z)-2=\prod_{\mathrm{k}=1}^{N}\left(2 z-2 \cos \frac{2 \pi \mathrm{k}}{N}\right)
$$

where the Chebyshev polynomial of the first kind is

$$
T_{N}(\cos \theta)=\cos (n \theta) \text {. }
$$

We conclude that

$$
s^{C_{2 N}}=2(c d)^{N}\left\{T_{2 N}(y)-1\right\} .
$$

We can also derive a formula for the half ring by using Eq. (7.4) in conjunction with the standard formula

$$
T_{2 N}(z)=T_{N}\left(2 z^{2}-1\right)
$$

to give

$$
s^{C_{2 N}}=2(c d)^{N}\left(T_{N}(x)-1\right),
$$

where we have used the definition of $x$ in Eq. (3.5).

The characteristic polynomial, $s^{C_{2 N}^{f}}(E)$, for the graph $C_{2 N}^{f}(a, b \mid c, d)$ is

$$
s^{C_{2 N}^{f}}=\prod_{\mathrm{k}=1}^{N}\left(E-E_{\mathrm{k}+}^{\mathrm{C}^{f}}\right)\left(E-E_{\mathrm{k}-}^{\mathrm{C}^{f}}\right)=\prod_{\mathrm{k}=1}^{N}\left(2 c d x^{2}-2 c d \cos \frac{\pi(2 \mathrm{k}-1)}{N}\right) .
$$

Expressing the cosine in terms of the half-angle, we find that

$$
s^{C_{2 N}^{f}}=(4 c d)^{N} \prod_{\mathrm{k}=1}^{N}\left(y^{2}-\cos ^{2} \frac{\pi(2 \mathrm{k}-1)}{2 N}\right)=4(-c d)^{N} T_{N}(y) T_{N}(-y),
$$

where we have used $y$ as in Eq. (3.10), and the well-known relation

$$
T_{N}(z)=2^{N-1} \prod_{\mathrm{k}=1}^{N}\left\{z-\cos \left(\frac{\pi(2 \mathrm{k}-1)}{2 N}\right)\right\}
$$

The product formula

$$
2 T_{N}^{2}(z)=T_{2 N}(z)+1
$$

and the parity of the Chebychev polynomials gives the final 'full-ring' expression

$$
s^{C_{2 N}^{f}}=2(c d)^{N}\left(T_{2 N}(y)+1\right) .
$$

We can also derive a formula for the half ring using the transformation in Eq. (7.5), to give

$$
s^{C_{2 N}^{f}}=2(c d)^{N}\left(T_{N}(x)+1\right) .
$$

The remaining structural polynomials can be deduced using the fact that removal of a vertex from a cycle gives rise to a path, and we have already derived the characteristic polynomials of even and odd vertex paths in Section 3. There are two kinds of vertex in 
our alternating cycles, odd-numbered vertices with weight $a$, and even-numbered vertices with weight $b$. When one of these vertices is removed we create a path of length $2 N-1$. The equations for the diagonal parts of $\jmath_{\mathrm{pq}}$ for $C_{2 N}(a, b \mid c, d)$ are:

$$
\begin{aligned}
J_{2 \mathrm{p}, 2 \mathrm{p}}^{C_{2 N}} & =(c d)^{N-1}(E-a) U_{N-1}(x), \\
J_{2 \mathrm{p}+1,2 \mathrm{p}+1}^{C_{2 N}} & =(c d)^{N-1}(E-b) U_{N-1}(x) .
\end{aligned}
$$

The formulae for the off-diagonal $\jmath$ polynomials can be deduced using Eq. (1.6), and by noting that if we remove a second vertex, then we form two (or possibly one) paths. This requires some trivial but lengthy trigonometry. The results are

$$
\begin{aligned}
J_{2 \mathrm{p}, 2 \mathrm{p}+2 \mathrm{q}}^{C_{2 N}} & =(E-a)(c d)^{N-1}\left\{U_{N-\mathrm{q}-1}(x)+U_{\mathrm{q}-1}(x)\right\}, \\
J_{2 \mathrm{p}+1,2 \mathrm{p}+2 \mathrm{q}+1}^{C_{2 N}} & =(E-b)(c d)^{N-1}\left\{U_{N-\mathrm{q}-1}(x)+U_{\mathrm{q}-1}(x)\right\}, \\
J_{2 \mathrm{p}+1,2 \mathrm{p}+2 \mathrm{q}+2}^{C_{2 N}} & =(c d)^{N-1}\left\{\widetilde{U}_{N-\mathrm{q}-1}(x ; c, d)+\widetilde{U}_{\mathrm{q}}(x ; d, c)\right\} .
\end{aligned}
$$

Note that the formulae do not depend upon p, but only upon q, the offset along the ring. The results for the flipped cycle can be calculated in the same manner. The diagonal $\jmath$ quantities are identical to those for the cycle. This is because the deletion of a vertex creates a chain, and flipped edges can be removed from a tree using an orthogonal transformation. Hence,

$$
\begin{aligned}
J_{2 \mathrm{p}, 2 \mathrm{p}}^{C^{f}} & =(c d)^{N-1}(E-a) U_{N-1}(x), \\
J_{2 \mathrm{p}+1,2 \mathrm{p}+1}^{f} & =(c d)^{N-1}(E-b) U_{N-1}(x),
\end{aligned}
$$

and further

$$
\begin{aligned}
J_{2 \mathrm{p}, 2 \mathrm{p}+2 \mathrm{q}}^{C^{f}} & =(E-a)(c d)^{N-1}\left\{U_{N-\mathrm{q}-1}(x)-U_{\mathrm{q}-1}(x)\right\}, \\
J_{2 \mathrm{p}+1,2 \mathrm{p}+2 \mathrm{q}+1}^{C_{2 N}^{f}} & =(E-b)(c d)^{N-1}\left\{U_{N-\mathrm{q}-1}(x)-U_{\mathrm{q}-1}(x)\right\}, \\
J_{2 \mathrm{p}+1,2 \mathrm{p}+2 \mathrm{q}+2}^{C_{2 N}^{f}} & =(c d)^{N-1}\left\{\widetilde{U}_{N-\mathrm{q}-1}(x ; c, d)-\widetilde{U}_{\mathrm{q}}(x ; d, c)\right\} .
\end{aligned}
$$

The changes in sign between Eqs. (7.14) and (7.16) arise because of the sign change between Eqs. (7.6) and (7.12).

\section{Alternating treadmills}

Treadmills are cyclic ladders. We consider a $4 N$-vertex treadmill, $T_{4 N}(a, b \mid c, d)$, with rung edge weights $(a, b)$, and backbone weights $(c, d)$ displayed in Fig. 5. We shall also consider a related treadmill, namely the 'flipped' treadmill, $T_{4 N}^{f}(a, b \mid c, d)$, which has a pair of symmetrically related backbone edges with weights having a changed sign. We also include in our discussion the Möbius treadmill, $T_{4 N}^{M}(a, b \mid c, d)$, which has a pair of crossed edges connecting top and bottom rings. There is also the flipped Möbius treadmill, $T_{4 N}^{M^{f}}(a, b \mid c, d)$, which has a pair of crossed bottom and top edges with weights having a changed sign. All of these treadmills possess the same involution symmetry, and hence can be treated in the same way. 


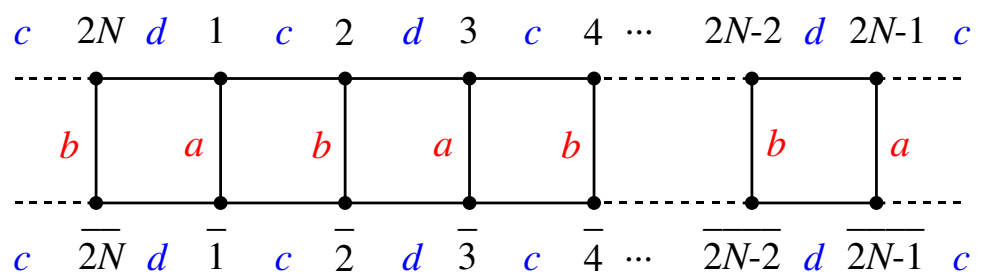

Figure 5: A treadmill, $T_{4 N}(a, b \mid c, d)$, with $4 N$ vertices and alternating rung $(a, b)$ and ring edge weights $(c, d)$.

\subsection{The treadmill $T_{4 N}(a, b \mid c, d)$}

This system has an involution symmetry based upon exchange of vertices attached to the ends of rungs. The methodology proceeds in exactly the same way as for the ladder example in Section 4 . Hence, the $4 \times 4$ blocks of vertices (top ring odd, top ring even, bottom ring odd, and bottom ring even) produce the adjacency matrix

$$
\left(\begin{array}{cccc}
\mathbf{0} & \mathbf{B}^{\mathrm{C}} & a \mathbf{1} & \mathbf{0} \\
\left(\mathbf{B}^{\mathrm{C}}\right)^{\mathrm{T}} & \mathbf{0} & \mathbf{0} & b \mathbf{1} \\
a \mathbf{1} & \mathbf{0} & \mathbf{0} & \mathbf{B}^{\mathrm{C}} \\
\mathbf{0} & b \mathbf{1} & \left(\mathbf{B}^{\mathrm{C}}\right)^{\mathrm{T}} & \mathbf{0}
\end{array}\right)
$$

where $\mathbf{B}^{\mathrm{C}}$ is the same matrix as in Eq. (6.2). This matrix can be block-diagonalised by an orthogonal transformation of the form

$$
\left(\begin{array}{cr}
\frac{1}{\sqrt{2}} \mathbf{1}_{2 N} & \frac{1}{\sqrt{2}} \mathbf{1}_{2 N} \\
\frac{1}{\sqrt{2}} \mathbf{1}_{2 N} & -\frac{1}{\sqrt{2}} \mathbf{1}_{2 N}
\end{array}\right) .
$$

The adjacency matrix after the transformation is:

$$
\left(\begin{array}{cccc}
a \mathbf{1} & \mathbf{B}^{\mathrm{C}} & \mathbf{0} & \mathbf{0} \\
\left(\mathbf{B}^{\mathrm{C}}\right)^{\mathrm{T}} & b \mathbf{1} & \mathbf{0} & \mathbf{0} \\
\mathbf{0} & \mathbf{0} & -a \mathbf{1} & \mathbf{B}^{\mathrm{C}} \\
\mathbf{0} & \mathbf{0} & \left(\mathbf{B}^{\mathrm{C}}\right)^{\mathrm{T}} & -b \mathbf{1}
\end{array}\right)=\left(\begin{array}{cc}
\mathbf{A}^{\mathrm{C}} & \mathbf{0} \\
\mathbf{0} & \mathbf{A}^{\overline{\mathrm{C}}}
\end{array}\right)
$$

in which $\mathbf{A}^{\mathrm{C}}$ is the adjacency matrix of $C_{2 N}(a, b \mid c, d)$ as shown in Eq. (6.1), and $\mathbf{A}^{\overline{\mathrm{C}}}$ is the adjacency matrix of $C_{2 N}(-a,-b \mid c, d)$, the ring with vertex weights of opposite sign. It follows that we can use the results of Section 6 to derive expressions for all relevant quantities. The eigenvalues for $T_{4 N}(a, b \mid c, d)$ are therefore

$$
\begin{aligned}
& E_{\mathrm{k} \pm}^{\mathrm{T}(s)}=E_{\mathrm{k} \pm}^{\mathrm{C}}, \\
& E_{\mathrm{k} \pm}^{\mathrm{T}(a)}=E_{\mathrm{k} \pm}^{\overline{\mathrm{C}}} \quad \text { for } \mathrm{k}=1,2, \ldots, N
\end{aligned}
$$

where $E^{\mathrm{C}}$ is the expression given in Eq. (6.10) for the eigenvalues of the cycle $C_{2 N}(a, b \mid$ $c, d)$, and $E^{\overline{\mathrm{C}}}$ refers to the eigenvalues of the cycle $C_{2 N}(-a,-b \mid c, d)$ with reversed vertex 
weights. The eigenvectors for the treadmill can be placed in a vector $\mathbf{c}^{\mathrm{T}}$, with entries

$$
\begin{aligned}
& c_{\mathrm{pk} \pm}^{\mathrm{T}(s)}=c_{\overline{\mathrm{pk}} \pm}^{\mathrm{T}(s)}=\frac{1}{\sqrt{2}} c_{\mathrm{pk} \pm}^{C}, \\
& c_{\mathrm{pk} \pm}^{\mathrm{T}(a)}=-c_{\overline{\mathrm{pk}} \pm}^{\mathrm{T}(a)}=\frac{1}{\sqrt{2}} c_{\mathrm{pk} \pm}^{\bar{C}},
\end{aligned}
$$

where the superscripts $(s)$, and $(a)$ indicate the symmetric and antisymmetric eigenvectors, respectively.

\subsection{Flipped treadmills, $T_{4 N}^{f}(a, b \mid c, d)$}

Flipped treadmills can be obtained by simply changing the sign of a pair of symmetrically positioned edges on the top and bottom rings. We shall take edges $(1,2 N)$ and $(\overline{1}, \overline{2 N})$ to have weights $-d$, without loss of generality, since a series of orthogonal transformations can move the flipped pair of edges to any position around the rings. We use the same diagonalization methodology as for the standard treadmill, whence the adjacency matrix is

$$
\left(\begin{array}{cccc}
a \mathbf{1} & \mathbf{B}^{\mathrm{C}^{f}} & \mathbf{0} & \mathbf{0} \\
\left(\mathbf{B}^{\mathrm{C}^{f}}\right)^{\mathrm{T}} & b \mathbf{1} & \mathbf{0} & \mathbf{0} \\
\mathbf{0} & \mathbf{0} & -a \mathbf{1} & \mathbf{B}^{\mathrm{C}^{f}} \\
\mathbf{0} & \mathbf{0} & \left(\mathbf{B}^{\mathrm{C}^{f}}\right)^{\mathrm{T}} & -b \mathbf{1}
\end{array}\right)=\left(\begin{array}{cc}
\mathbf{A}^{\mathrm{C}^{f}} & \mathbf{0} \\
\mathbf{0} & \mathbf{A}^{\overline{\mathrm{C}}^{f}}
\end{array}\right)
$$

where the matrix $\mathbf{B}^{\mathrm{C}^{f}}$ is shown in Eq. (6.15), $\mathbf{A}^{\mathrm{C}^{f}}$ is the adjacency matrix of $C_{2 N}^{f}(a, b \mid$ $c, d)$ as shown in Eq. (6.14), and $\mathbf{A}^{\overline{\mathrm{C}}^{f}}$ is the adjacency matrix of $C_{2 N}^{f}(-a,-b \mid c, d)$, the ring with vertex weights of opposite sign. The eigenvalues for $T_{4 N}^{f}(a, b \mid c, d)$ are thus

$$
\begin{aligned}
& E_{\mathrm{k} \pm}^{\mathrm{T}^{f}(s)}=E_{\mathrm{k} \pm}^{\mathrm{C}^{f}}, \\
& E_{\mathrm{k} \pm}^{\mathrm{T}^{f}(a)}=E_{\mathrm{k} \pm}^{\overline{\mathrm{C}}^{f}}
\end{aligned} \quad \text { for } \mathrm{k}=1,2, \ldots, N,
$$

where $E_{\mathrm{k} \pm}^{\mathrm{C}^{f}}$, given in Eq. (6.20), is an eigenvalue of $C_{2 N}^{f}(a, b \mid c, d)$, and $E_{\mathrm{k} \pm}^{\overline{\mathrm{C}}^{f}}$ is an eigenvalue of $C_{2 N}^{f}(-a,-b \mid c, d)$.

The eigenfunction entries can be written as

$$
\begin{aligned}
& c_{\mathrm{pk} \pm}^{\mathrm{T}^{f}(s)}=c_{\overline{\mathrm{pk}} \pm}^{\mathrm{T}^{f}(s)}=\frac{1}{\sqrt{2}} c_{\mathrm{pk} \pm}^{C^{f}} \\
& c_{\mathrm{pk} \pm}^{\mathrm{T}^{f}(a)}=-c_{\overline{\mathrm{pk}} \pm}^{\mathrm{T}^{f}(a)}=\frac{1}{\sqrt{2}} c_{\mathrm{pk} \pm}^{\bar{C}^{f}}
\end{aligned}
$$

with $2 N$ eigenvectors in the symmetric and the antisymmetric blocks (i.e. $\mathrm{k}=1,2, \ldots, N)$.

\subsection{Möbius treadmills, $T_{4 N}^{M}(a, b \mid c, d)$}

The Möbius treadmill has the same involution symmetry as the other treadmills defined in Section 8. Using the treadmill block diagonalisation procedure, the adjacency matrix 


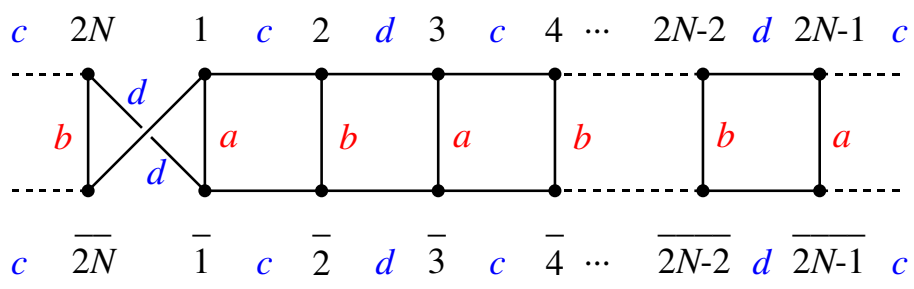

Figure 6: A Möbius treadmill, $T_{4 N}^{M}(a, b \mid c, d)$, with $4 N$ vertices and alternating rung $(a, b)$ and ring edge weights $(c, d)$.

becomes

$$
\left(\begin{array}{cccc}
a \mathbf{1} & \mathbf{B}^{\mathrm{C}} & \mathbf{0} & \mathbf{0} \\
\left(\mathbf{B}^{\mathrm{C}}\right)^{\mathrm{T}} & b \mathbf{1} & \mathbf{0} & \mathbf{0} \\
\mathbf{0} & \mathbf{0} & -a \mathbf{1} & \mathbf{B}^{\mathrm{C}^{f}} \\
\mathbf{0} & \mathbf{0} & \left(\mathbf{B}^{\mathrm{C}^{f}}\right)^{\mathrm{T}} & -b \mathbf{1}
\end{array}\right)=\left(\begin{array}{cc}
\mathbf{A}^{\mathrm{C}} & \mathbf{0} \\
\mathbf{0} & \mathbf{A}^{\overline{\mathrm{C}}^{f}}
\end{array}\right),
$$

which has the structure of $C_{2 N}(a, b \mid c, d)$ in the top block, and $C_{2 N}^{f}(-a,-b \mid c, d)$ in the lower one. The eigenvalues for $T_{4 N}^{M}(a, b \mid c, d)$ are thus

$$
\begin{aligned}
& E_{\mathrm{k} \pm}^{\mathrm{T}^{M}(s)}=E_{\mathrm{k} \pm}^{\mathrm{C}}, \\
& E_{\mathrm{k} \pm}^{\mathrm{T}^{M}(a)}=E_{\mathrm{k} \pm}^{\overline{\mathrm{C}}^{f}} \quad \text { for } \mathrm{k}=1,2, \ldots, N .
\end{aligned}
$$

The eigenfunction entries can be written as

$$
\begin{gathered}
c_{\mathrm{pk} \pm}^{\mathrm{T}^{M}(s)}=c_{\overline{\mathrm{pk}} \pm}^{\mathrm{T}^{M}(s)}=\frac{1}{\sqrt{2}} c_{\mathrm{pk} \pm}^{C}, \\
c_{\mathrm{pk} \pm}^{\mathrm{T}^{M}(a)}=-c_{\overline{\mathrm{pk}} \pm}^{\mathrm{T}^{M}(a)}=\frac{1}{\sqrt{2}} c_{\mathrm{pk} \pm}^{C^{f}},
\end{gathered}
$$

with $2 N$ eigenvectors in the symmetric and the antisymmetric blocks.

8.4 Flipped Möbius treadmills, $T_{4 N}^{M^{f}}(a, b \mid c, d)$

The block diagonalisation procedure gives the adjacency matrix

$$
\left(\begin{array}{cccc}
a \mathbf{1} & \left(\mathbf{B}^{\mathrm{C}^{f}}\right)^{\mathrm{T}} & \mathbf{0} & \mathbf{0} \\
\mathbf{B}^{\mathrm{C}^{f} T} & b \mathbf{1} & \mathbf{0} & \mathbf{0} \\
\mathbf{0} & \mathbf{0} & -a \mathbf{1} & \mathbf{B}^{\mathrm{C}} \\
\mathbf{0} & \mathbf{0} & \left(\mathbf{B}^{\mathrm{C}}\right)^{\mathrm{T}} & -b \mathbf{1}
\end{array}\right)=\left(\begin{array}{cc}
\mathbf{A}^{\mathrm{C}^{f}} & \mathbf{0} \\
\mathbf{0} & \mathbf{A}^{\overline{\mathrm{C}}}
\end{array}\right)
$$

which has the structure of $C_{2 N}^{f}(a, b \mid c, d)$ in the top block, and $C_{2 N}(-a,-b \mid c, d)$ in the lower. The eigenfunction entries, in this case, are

$$
\begin{gathered}
c_{\mathrm{pk} \pm}^{\mathrm{T}^{M^{f}}(s)}=c_{\overline{\mathrm{pk}} \pm}^{\mathrm{T}^{M^{f}}(s)}=\frac{1}{\sqrt{2}} c_{\mathrm{pk} \pm}^{C^{f}}, \\
c_{\mathrm{pk} \pm}^{\mathrm{T}^{M^{f}}(a)}=-c_{\overline{\mathrm{pk}} \pm}^{\mathrm{T}^{M^{f}}(a)}=\frac{1}{\sqrt{2}} c_{\mathrm{pk} \pm}^{\bar{C}} .
\end{gathered}
$$




\section{Structural polynomials of alternating treadmills}

The characteristic polynomial, $s^{T_{4 N}}(E)$, can be written immediately because we have been able to split the secular equations into two annulene terms, $C_{2 N}(a, b \mid c, d)$ and $C_{2 N}(-a,-b \mid c, d)$, to give

$$
\begin{aligned}
s^{T_{4 N}} & =s\left(C_{2 N}(a, b \mid c, d), E\right) s\left(C_{2 N}(-a,-b \mid c, d), E\right) \\
& =4(c d)^{2 N}\left(T_{N}(x)-1\right)\left(T_{N}(\bar{x})-1\right),
\end{aligned}
$$

with $x$ and $\bar{x}$ defined in Eqs. (3.5) and (5.2). We can also write

$$
s^{T_{4 N}}=4(c d)^{2 N}\left(T_{2 N}(y)-1\right)\left(T_{2 N}(\bar{y})-1\right)
$$

with $y$ and $\bar{y}$ defined in Eqs. (3.10) and (5.4). The factoring of the secular problem for the treadmill allows the characteristic polynomials to be written as a product of the characteristic polynomials of $C_{2 N}(a, b \mid c, d)$ and $C_{2 N}(-a,-b \mid c, d)$.

The other structural polynomials can be obtained using the same logic as in Section 5,

$$
\begin{aligned}
J_{\mathrm{pq}}^{T_{4 N}} & =\frac{1}{2}\left(\jmath\left(C_{2 N}(a, b \mid c, d), E\right) s\left(C_{2 N}(-a,-b \mid c, d), E\right)\right. \\
& \left.+s\left(C_{2 N}(a, b \mid c, d), E\right) \jmath\left(C_{2 N}(-a,-b \mid c, d), E\right)\right) .
\end{aligned}
$$

It follows that, for pairs of indices on the same ring,

$$
\begin{aligned}
& J_{2 \mathrm{p}, 2 \mathrm{p}+2 \mathrm{q}}^{T_{4}}=(c d)^{2 N-1}\left\{(E-a)\left[U_{N-\mathrm{q}-1}(x)+U_{\mathrm{q}-1}(x)\right]\left[T_{N}(\bar{x})-1\right]\right. \\
&+\left.(E+a)\left[U_{N-\mathrm{q}-1}(\bar{x})+U_{\mathrm{q}-1}(\bar{x})\right]\left[T_{N}(x)-1\right]\right\}, \\
& J_{2 \mathrm{p}+1,2 \mathrm{p}+2 \mathrm{q}+1}^{T_{4 N}}=(c d)^{2 N-1}\left\{(E-b)\left[U_{N-\mathrm{q}-1}(x)+U_{\mathrm{q}-1}(x)\right]\left[T_{N}(\bar{x})-1\right]\right. \\
&\left.+(E+a)\left[U_{N-\mathrm{q}-1}(\bar{x})+U_{\mathrm{q}-1}(\bar{x})\right]\left[T_{N}(x)-1\right]\right\}, \\
& J_{2 \mathrm{p}+1,2 \mathrm{p}+2 \mathrm{q}+2}^{T_{4 N}}=(c d)^{2 N-1}\left\{\left[U_{N-\mathrm{q}-1}(x)+U_{\mathrm{q}}(x)\right]\left[T_{N}(\bar{x})-1\right]\right. \\
&\left.+\left[U_{N-\mathrm{q}-1}(\bar{x})+U_{\mathrm{q}}(\bar{x})\right]\left[T_{N}(x)-1\right]\right\},
\end{aligned}
$$

and for pairs of indices on different rings,

$$
\begin{aligned}
J_{2 \mathrm{p}, 2 \overline{\mathrm{p}}+2 \overline{\mathrm{q}}}^{T_{4 N}}=(c d)^{2 N-1} & \left\{(E-a)\left[U_{N-\mathrm{q}-1}(x)+U_{\mathrm{q}-1}(x)\right]\left[T_{N}(\bar{x})-1\right]\right. \\
- & \left.(E+b)\left[U_{N-\mathrm{q}-1}(\bar{x})+U_{\mathrm{q}-1}(\bar{x})\right]\left[T_{N}(x)-1\right]\right\}, \\
T_{4 N} & \left\{(E-b)\left[U_{N-\mathrm{q}-1}(x)+U_{\mathrm{q}-1}(x)\right]\left[T_{N}(\bar{x})-1\right]\right. \\
T_{2 \mathrm{p}+1,2 \overline{\mathrm{p}}+2 \overline{\mathrm{q}}+1}=(c d)^{2 N-1} & \left.(E+b)\left[U_{N-\mathrm{q}-1}(\bar{x})+U_{\mathrm{q}-1}(\bar{x})\right]\left[T_{N}(x)-1\right]\right\}, \\
- & \left.(E)+U_{\mathrm{q}}(x)\right]\left[T_{N}(\bar{x})-1\right] \\
J_{2 \mathrm{p}+1,2 \overline{\mathrm{p}}+2 \overline{\mathrm{q}}+2}^{T_{4 N}}=(c d)^{2 N-1}\left\{\left[U_{N-\mathrm{q}-1}(x)+U_{N}(x)-1\right]\right\}, & {\left[U_{N-\mathrm{q}-1}(\bar{x})+U_{\mathrm{q}}(\bar{x})\right]\left[T_{N}(x)\right.}
\end{aligned}
$$

where we observe the expected sign change arising from the antisymmetry.

\subsection{Structural polynomials of $T_{4 N}^{f}(a, b \mid c, d)$}

The characteristic polynomial, $s^{T_{4 N}^{f}}(E)$, can be written immediately as we have been able to split the secular equations into contributions from two flipped annulenes, $C_{2 N}^{f}(a, b \mid c, d)$ 
and $C_{2 N}^{f}(-a,-b \mid c, d)$, to give

$$
\begin{aligned}
s^{T_{4 N}^{f}} & =s\left(C_{2 N}^{f}(a, b \mid c, d), E\right) s\left(C_{2 N}^{f}(-a,-b \mid c, d), E\right) \\
& =4(c d)^{2 N}\left(T_{N}(x)+1\right)\left(T_{N}(\bar{x})+1\right)
\end{aligned}
$$

with $x$ and $\bar{x}$ defined in Eqs. (3.5) and (5.2). We can also write a product form

$$
s^{T_{4 N}^{f}}=4(c d)^{2 N}\left(T_{2 N}(y)+1\right)\left(T_{2 N}(\bar{y})+1\right)
$$

with $y$ and $\bar{y}$ defined in Eqs. (3.10) and (5.4).

The $\jmath$ structural polynomials can be obtained using the same logic as in Section 5:

$$
\begin{aligned}
\jmath_{\mathrm{pq}}^{T_{4 N}^{f}} & =\frac{1}{2}\left(\jmath\left(C_{2 N}^{f}(a, b \mid c, d), E\right) s\left(C_{2 N}^{f}(-a,-b \mid c, d), E\right)\right. \\
& \left.+s\left(C_{2 N}^{f}(a, b \mid c, d), E\right) \jmath\left(C_{2 N}^{f}(-a,-b \mid c, d), E\right)\right),
\end{aligned}
$$

so that, for indices on the same ring,

$$
\begin{aligned}
& J_{2 \mathrm{p}, 2 \mathrm{p}+2 \mathrm{q}}^{T_{f}^{f}}=(c d)^{2 N-1}\left\{(E-a)\left[U_{N-\mathrm{q}-1}(x)-U_{\mathrm{q}-1}(x)\right]\left[T_{N}(\bar{x})+1\right]\right. \\
&+\left.(E+a)\left[U_{N-\mathrm{q}-1}(\bar{x})-U_{\mathrm{q}-1}(\bar{x})\right]\left[T_{N}(x)+1\right]\right\}, \\
& J_{2 \mathrm{p}+1,2 \mathrm{p}+2 \mathrm{q}+1}=(c d)^{2 N-1}\left\{(E-b)\left[U_{N-\mathrm{q}-1}(x)-U_{\mathrm{q}-1}(x)\right]\left[T_{N}(\bar{x})+1\right]\right. \\
&\left.+(E+a)\left[U_{N-\mathrm{q}-1}(\bar{x})-U_{\mathrm{q}-1}(\bar{x})\right]\left[T_{N}(x)+1\right]\right\}, \\
& T_{2 \mathrm{p}+1,2 \mathrm{p}+2 \mathrm{q}+2}^{f}=(c d)^{2 N-1}\left\{\left[U_{N-\mathrm{q}-1}(x)-U_{\mathrm{q}}(x)\right]\left[T_{N}(\bar{x})+1\right]\right. \\
&\left.+\left[U_{N-\mathrm{q}-1}(\bar{x})-U_{\mathrm{q}}(\bar{x})\right]\left[T_{N}(x)+1\right]\right\},
\end{aligned}
$$

and, for indices on different rings,

$$
\begin{aligned}
J_{2 \mathrm{p}, 2 \overline{\mathrm{p}}+2 \overline{\mathrm{q}}}^{f}=(c d)^{2 N-1} & \left\{(E-a)\left[U_{N-\mathrm{q}-1}(x)-U_{\mathrm{q}-1}(x)\right]\left[T_{N}(\bar{x})+1\right]\right. \\
- & \left.(E+b)\left[U_{N-\mathrm{q}-1}(\bar{x})-U_{\mathrm{q}-1}(\bar{x})\right]\left[T_{N}(x)+1\right]\right\}, \\
T_{2 \mathrm{p}+1,2 \overline{\mathrm{p}}+2 \overline{\mathrm{q}}+1}^{f}=(c d)^{2 N-1}\left\{(E-b)\left[U_{N-\mathrm{q}-1}(x)-U_{\mathrm{q}-1}(x)\right]\left[T_{N}(\bar{x})+1\right]\right. & \\
- & \left.(E+b)\left[U_{N-\mathrm{q}-1}(\bar{x})-U_{\mathrm{q}-1}(\bar{x})\right]\left[T_{N}(x)+1\right]\right\}, \\
T_{4 N}^{f} & \left\{\left[U_{N-\mathrm{q}-1}(x)-U_{\mathrm{q}}(x)\right]\left[T_{N}(\bar{x})+1\right]\right. \\
T_{2 \mathrm{p}+1,2 \overline{\mathrm{p}}+2 \overline{\mathrm{q}}+2}^{f}=(c d)^{2 N-1} & \left.\left\{U_{N-\mathrm{q}-1}(\bar{x})-U_{\mathrm{q}}(\bar{x})\right]\left[T_{N}(x)+1\right]\right\} .
\end{aligned}
$$

The differences between equations for plain (c.f. Eqs. (9.4) and (9.5)) and flipped treadmills occur because of the sign changes arising from edge flips. These affect all terms inside the square brackets because symmetry divides the adjacency matrix into two flipped $\operatorname{rings} C_{2 N}^{f}$.

\subsection{Structural polynomials of $T_{4 N}^{M}(a, b \mid c, d)$}

The characteristic polynomial, $s^{T_{4 N}^{M}}(E)$, can be written immediately as we have been able to split the secular equations into contributions from two annulenes, $C_{2 N}(a, b \mid c, d)$ and 
$C_{2 N}^{f}(-a,-b \mid c, d)$, to give

$$
\begin{aligned}
s^{T_{4 N}^{M}} & =s\left(C_{2 N}(a, b \mid c, d), E\right) s\left(C_{2 N}^{f}(-a,-b \mid c, d), E\right) \\
& =4(c d)^{2 N}\left(T_{N}(x)-1\right)\left(T_{N}(\bar{x})+1\right),
\end{aligned}
$$

with $x$ and $\bar{x}$ defined in Eqs. (3.5) and (5.2). We can also write

$$
s^{T_{4 N}^{M}}=4(c d)^{2 N}\left(T_{2 N}(y)-1\right)\left(T_{2 N}(\bar{y})+1\right),
$$

with $y$ and $\bar{y}$ defined in Eqs. (3.10) and (5.4).

The $\jmath$ structural polynomials can be obtained using the same logic as in Section 5,

$$
\begin{aligned}
\jmath_{\mathrm{pq}}^{T_{4 N}^{M}} & =\frac{1}{2}\left(\jmath\left(C_{2 N}(a, b \mid c, d), E\right) s\left(C_{2 N}^{f}(-a,-b \mid c, d), E\right)\right. \\
& \left.+s\left(C_{2 N}(a, b \mid c, d), E\right) \jmath\left(C_{2 N}^{f}(-a,-b \mid c, d), E\right)\right),
\end{aligned}
$$

so that, for indices on the same ring,

$$
\begin{aligned}
& T_{4 N}^{M} \\
& T_{2 \mathrm{p}, 2 \mathrm{p}+2 \mathrm{q}}=(c d)^{2 N-1}\left\{(E-a)\left[U_{N-\mathrm{q}-1}(x)+U_{\mathrm{q}-1}(x)\right]\left[T_{N}(\bar{x})+1\right]\right. \\
&+\left.(E+a)\left[U_{N-\mathrm{q}-1}(\bar{x})-U_{\mathrm{q}-1}(\bar{x})\right]\left[T_{N}(x)-1\right]\right\}, \\
& T_{2 N}^{M}\left\{(E-b)\left[U_{N+\mathrm{q}-1}(x)+U_{\mathrm{q}-1}(x)\right]\left[T_{N}(\bar{x})+1\right]\right. \\
&+\left.(E+a)\left[U_{N-\mathrm{q}-1}(\bar{x})-U_{\mathrm{q}-1}(\bar{x})\right]\left[T_{N}(x)-1\right]\right\}, \\
& J_{2 \mathrm{p}+1,2 \mathrm{p}+2 \mathrm{q}+1}=(c d)^{2 N-1}{ }^{M}\left\{\left[U_{N-\mathrm{q}-1}(x)+U_{\mathrm{q}}(x)\right]\left[T_{N}(\bar{x})+1\right]\right. \\
&+ {\left.\left[U_{N-\mathrm{q}-1}(\bar{x})-U_{\mathrm{q}}(\bar{x})\right]\left[T_{N}(x)-1\right]\right\}, }
\end{aligned}
$$

whilst for indices on different rings,

$$
\begin{aligned}
& J_{2 \mathrm{p}, 2 \overline{\mathrm{p}}+2 \overline{\mathrm{q}}}^{M}=(c d)^{2 N-1}\left\{(E-a)\left[U_{N-\mathrm{q}-1}(x)+U_{\mathrm{q}-1}(x)\right]\left[T_{N}(\bar{x})+1\right]\right. \\
&-\left.(E+b)\left[U_{N-\mathrm{q}-1}(\bar{x})-U_{\mathrm{q}-1}(\bar{x})\right]\left[T_{N}(x)-1\right]\right\}, \\
&\left\{(E-b)\left[U_{N-\mathrm{q}-1}(x)+U_{\mathrm{q}-1}(x)\right]\left[T_{N}(\bar{x})+1\right]\right. \\
&\left.J^{T_{4 N}^{M}}\right)_{2 \mathrm{p}+1,2 \overline{\mathrm{p}}+2 \overline{\mathrm{q}}+1}=(c d)^{2 N-1}\left.(E+b)\left[U_{N-\mathrm{q}-1}(\bar{x})-U_{\mathrm{q}-1}(\bar{x})\right]\left[T_{N}(x)-1\right]\right\}, \\
& J_{2 \mathrm{p}+1,2 \overline{\mathrm{p}}+2 \overline{\mathrm{q}}+2}^{T_{1}^{M}}=(c d)^{2 N-1}\left\{\left[U_{N-\mathrm{q}-1}(x)+U_{\mathrm{q}}(x)\right]\left[T_{N}(\bar{x})+1\right]\right. \\
&\left.-\left[U_{N-\mathrm{q}-1}(\bar{x})-U_{\mathrm{q}}(\bar{x})\right]\left[T_{N}(x)-1\right]\right\} .
\end{aligned}
$$

The sign changes in this case affect only one of the sets of terms inside square brackets, because symmetry divides the adjacency matrix into contributions from one ring $C_{2 N}$ and one flipped ring $C_{2 N}^{f}$.

\subsection{Structural polynomials of $T_{4 N}^{M^{f}}(a, b \mid c, d)$}

The characteristic polynomial, $s_{4 N}^{T^{M^{f}}}(E)$, can be written immediately as we have been able to split the secular equations into contributions from annulenes, $C_{2 N}^{f}(a, b \mid c, d)$ and $C_{2 N}(-a,-b \mid c, d)$, to give

$$
\begin{aligned}
s_{4 N}^{T^{M^{f}}} & =s\left(C_{2 N}^{f}(a, b \mid c, d), E\right) s\left(C_{2 N}(-a,-b \mid c, d), E\right) \\
& =4(c d)^{2 N}\left(T_{N}(x)+1\right)\left(T_{N}(\bar{x})-1\right),
\end{aligned}
$$


with $x$ and $\bar{x}$ defined in Eqs. (3.5) and (5.2). We can also write

$$
s_{4 N}^{T^{M^{f}}}=4(c d)^{2 N}\left(T_{2 N}(y)+1\right)\left(T_{2 N}(\bar{y})-1\right)
$$

with $y$ and $\bar{y}$ defined in Eqs. (3.10) and (5.4).

The $\jmath$ structural polynomials can be obtained using the same logic as in Section 5,

$$
\begin{aligned}
\jmath_{\mathrm{pq}}^{T_{4 N}^{M^{f}}} & =\frac{1}{2}\left(\jmath\left(C_{2 N}^{f}(a, b \mid c, d), E\right) s\left(C_{2 N}(-a,-b \mid c, d), E\right)\right. \\
& \left.+s\left(C_{2 N}^{f}(a, b \mid c, d), E\right) \jmath\left(C_{2 N}(-a,-b \mid c, d), E\right)\right)
\end{aligned}
$$

so that, for indices on the same ring,

$$
\begin{aligned}
T_{4 N}^{T_{4}^{f}} \\
j_{2 \mathrm{p}, 2 \mathrm{p}+2 \mathrm{q}}=(c d)^{2 N-1}\left\{(E-a)\left[U_{N-\mathrm{q}-1}(x)-U_{\mathrm{q}-1}(x)\right]\left[T_{N}(\bar{x})-1\right]\right. \\
\left.+(E+a)\left[U_{N-\mathrm{q}-1}(\bar{x})+U_{\mathrm{q}-1}(\bar{x})\right]\left[T_{N}(x)+1\right]\right\}, \\
J_{2 \mathrm{p}+1,2 \mathrm{p}+2 \mathrm{q}+1}^{T^{f} f}=(c d)^{2 N-1}\left\{(E-b)\left[U_{N-\mathrm{q}-1}(x)-U_{\mathrm{q}-1}(x)\right]\left[T_{N}(\bar{x})-1\right)\right. \\
\left.+(E+a)\left[U_{N-\mathrm{q}-1}(\bar{x})+U_{\mathrm{q}-1}(\bar{x})\right]\left(T_{N}(x)+1\right]\right\}, \\
J_{2 \mathrm{p}+1,2 \mathrm{p}+2 \mathrm{q}+2}^{T^{f}}=(c d)^{2 N-1}\left\{\left[U_{N-\mathrm{q}-1}(x)-U_{\mathrm{q}}(x)\right]\left[T_{N}(\bar{x})-1\right]\right. \\
\left.+\left[U_{N-\mathrm{q}-1}(\bar{x})+U_{\mathrm{q}}(\bar{x})\right]\left[T_{N}(x)+1\right]\right\},
\end{aligned}
$$

and for indices on different rings,

$$
\begin{aligned}
& j_{2 \mathrm{p}, 2 \overline{\mathrm{p}}+2 \overline{\mathrm{q}}}^{T_{4 N}^{M^{f}}}=(c d)^{2 N-1}\left\{(E-a)\left[U_{N-\mathrm{q}-1}(x)-U_{\mathrm{q}-1}(x)\right]\left[T_{N}(\bar{x})-1\right]\right. \\
& \left.-(E+b)\left[U_{N-\mathrm{q}-1}(\bar{x})+U_{\mathrm{q}-1}(\bar{x})\right]\left[T_{N}(x)+1\right]\right\}, \\
& J_{2 \mathrm{p}+1,2 \overline{\mathrm{p}}+2 \overline{\mathrm{q}}+1}^{T_{4 N}^{M f}}=(c d)^{2 N-1}\left\{(E-b)\left[U_{N-\mathrm{q}-1}(x)-U_{\mathrm{q}-1}(x)\right]\left[T_{N}(\bar{x})-1\right)\right. \\
& \left.-(E+b)\left[U_{N-\mathrm{q}-1}(\bar{x})+U_{\mathrm{q}-1}(\bar{x})\right]\left[T_{N}(x)+1\right]\right\}, \\
& J_{2 \mathrm{p}+1,2 \overline{\mathrm{p}}+2 \overline{\mathrm{q}}+2}^{T_{4 N}^{M^{f}}}=(c d)^{2 N-1}\left\{\left[U_{N-\mathrm{q}-1}(x)-U_{\mathrm{q}}(x)\right]\left[T_{N}(\bar{x})-1\right]\right. \\
& \left.-\left[U_{N-\mathrm{q}-1}(\bar{x})+U_{\mathrm{q}}(\bar{x})\right]\left[T_{N}(x)+1\right]\right\} \text {. }
\end{aligned}
$$

The sign changes in this case affect only one of the sets of terms inside square brackets, because symmetry divides the adjacency matrix into a flipped ring $C_{2 N}^{f}$ and one plain ring $C_{2 N}$.

\section{Graphs derived from alternating ladders and treadmills}

A series of interesting graphs can be derived from our alternating ladders and treadmills by putting either $a=0$, or $b=0$. Some of the graphs that can be derived from ladders are shown in Fig. 7. Ladders with backbone chains with odd numbers of vertices lead to polyacenes with arms and legs, or to polyacenes themselves (Fig. 7(a) and 7(b)), by putting the first rung edge parameter or the second to zero. Even-vertex backbones give polyacenes with a single arm and leg, as shown in Fig. 7(c), whichever rung weight is set to zero. In the case of treadmills, it does not matter which rung weight is set to zero. In either case one obtains cyclic polyacenes. The appropriate formulae in Sections 4 and 8 for eigenvalues, eigenvectors and structural polynomials can be used in these cases. 


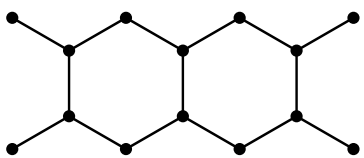

(a)

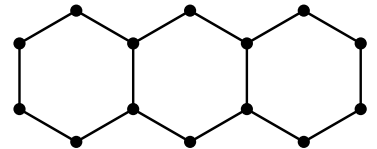

(b)

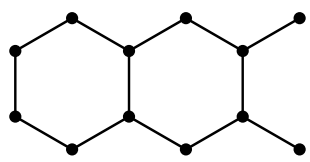

(c)

Figure 7: Graphs derived from ladders by zeroing rung parameters to zero: (a) $L_{14}(0,1$ | $1,1)$, with two 7-vertex backbone chains, $a=0$; (b) $L_{14}(0,1 \mid 1,1)$, with two 7-vertex backbone chains, $b=0$; (c) $L_{12}(1,0 \mid 1,1)$, with two 6-vertex backbone chains, $b=0$.

\section{Conclusions}

The algebraic development of structural polynomials reported here has been carried out in order to have exact results on which to base an elaboration of the theory of molecular conduction. The new formulae will allow us to treat $\pi$ distortivity and its effect on ballistic conduction through conjugated molecular frameworks, as predicted within the source-sink potential (SSP) approach, a model that has a very direct connection to graph theory. In the simplest picture, a molecular device consists of a molecule attached to two semi-infinite wires. Such a device can be modelled qualitatively by replacing the system by a graph in which the electronic interactions are replaced by a series of edge weights. Furthermore, the infinite wires can be replaced by source and sink vertices, with complex vertex weights modified to reproduce the physics of a current of electrons down the wires. The formula for the transmission, $T(E)$, of current of electrons through a device with energy $E$, can then be expressed in terms of four polynomials derived from the graph of the molecule. These polynomials can be chosen as the characteristic polynomial $s(E)$ of the graph itself, $\jmath_{\mathrm{pp}}(E), \jmath_{\mathrm{qq}}(E)$, and $\jmath_{\mathrm{pq}}(E)$ (c.f. Eqs. (1.1) and (1.2)), where vertices p and q of the molecule are attached to the source and sink vertices in the device.

The formulae we have obtained for ladders and the various forms of treadmills show that the structural polynomials can be written in a simple manner. We have shown that the existence of an involution allows the characteristic polynomials to be written neatly as a product of the characteristic polynomials of certain 'half' graphs comprising vertexweighted backbones. The remaining structural polynomials are also expressed in terms of half graphs, albeit in a slightly more complicated form.

Representation of the various structural polynomials in this 'factorised' form has advantages for understanding the structure of the spectrum and has implications for the physics of the transmission as a function of energy. The different sign patterns of the structural polynomials exhibited in, for example, the varieties of treadmill (flipped, Möbius, etc.) will have a profound effect on transmission, $T(E)$, as a function of the energy of the incoming electrons. In certain cases, for example, conduction is switched off for the whole range of accessible energies, $E$. A detailed account of the SSP modelling of conduction in systems represented by graphs with these exotic topologies will be published elsewhere.

\section{References}

[1] C. A. Coulson and G. S. Rushbrooke, Note on the method of molecular orbitals, Math. Proc. Cambridge Phil. Soc. 36 (1940), 193-200, doi:10.1017/s0305004100017163. 
[2] C. M. da Fonseca and J. Petronilho, Explicit inverses of some tridiagonal matrices, Linear Algebra Appl. 325 (2001), 7-21, doi:10.1016/s0024-3795(00)00289-5.

[3] M. Ernzerhof, Density functional theory of complex transition densities, J. Chem. Phys. 125 (2006), 124104, doi:10.1063/1.2348880.

[4] M. Ernzerhof, H. Bahmann, F. Goyer, M. Zhuang and P. Rocheleau, Electron transmission through aromatic molecules, J. Chem. Theory Comput. 2 (2006), 1291-1297, doi:10.1021/ ct600087c.

[5] M. Ernzerhof, M. Zhuang and P. Rocheleau, Side-chain effects in molecular electronic devices, J. Chem. Phys. 123 (2005), 134704, doi:10.1063/1.2049249.

[6] P. W. Fowler, Hückel spectra of Möbius $\pi$ systems, Phys. Chem. Chem. Phys. 4 (2002), 28782883, doi:10.1039/b201850k.

[7] P. W. Fowler, B. T. Pickup, T. Z. Todorova and W. Myrvold, A selection rule for molecular conduction, J. Chem. Phys. 131 (2009), 044104, doi:10.1063/1.3182849.

[8] G. H. Golub and C. F. Van Loan, Matrix Computations, Johns Hopkins Studies in the Mathematical Sciences, Johns Hopkins University Press, Baltimore, Maryland, 3rd edition, 1996.

[9] M. J. C. Gover, The eigenproblem of a tridiagonal 2-Toeplitz matrix, Linear Algebra Appl. 197, 198 (1994), 63-78, doi:10.1016/0024-3795(94)90481-2.

[10] I. S. Gradshteyn and I. M. Ryzhik, Table of Integrals, Series, and Products, Academic Press, Amsterdam, 8th edition, 2015.

[11] E. Heilbronner, Why do some molecules have symmetry different from that expected?, J. Chem. Educ. 66 (1989), 471-478, doi:10.1021/ed066p471.

[12] H. A. Jahn and E. Teller, Stability of polyatomic molecules in degenerate electronic states. I-Orbital degeneracy, Proc. R. Soc. A 161 (1937), 220-235, doi:10.1098/rspa.1937.0142.

[13] R. E. Peierls, Quantum Theory of Solids, Oxford Classic Texts in the Physical Sciences, Clarendon Press, Oxford, 1955, doi:10.1093/acprof:oso/9780198507819.001.0001.

[14] B. T. Pickup and P. W. Fowler, An analytical model for steady-state currents in conjugated systems, Chem. Phys. Lett. 459 (2008), 198-202, doi:10.1016/j.cplett.2008.05.062.

[15] B. T. Pickup, P. W. Fowler, M. Borg and I. Sciriha, A new approach to the method of sourcesink potentials for molecular conduction, J. Chem. Phys. 143 (2015), 194105, doi:10.1063/1. 4935716.

[16] B. T. Pickup, P. W. Fowler and I. Sciriha, A hückel source-sink-potential theory of pauli spin blockade in molecular electronic devices, J. Chem. Phys. 145 (2016), 204113, doi:10.1063/1. 4967957.

[17] I. Sciriha, M. Debono, M. Borg, P. W. Fowler and B. T. Pickup, Interlacing-extremal graphs, Ars Math. Contemp. 6 (2013), 261-278, http: / / amc-journal. eu/index.php/amc/ article/view/275.

[18] B. C. Shin, A formula for eigenpairs of certain symmetric tridiagonal matrices, Bull. Austral. Math. Soc. 55 (1997), 249-254, doi:10.1017/s0004972700033918.

[19] J. J. Sylvester, On the relation between the minor determinants of linearly equivalent quadratic functions, Philos. Mag. Ser. 41 (1851), 295-305, http://www.tandfonline.com/ doi/abs/10.1080/14786445108646735.

[20] M. Zhuang and M. Ernzerhof, Zero-voltage conductance of short gold nanowires, J. Chem. Phys. 120 (2004), 4921-4926, doi:10.1063/1.1644106. 